NATIONAL LABORATORY

\title{
ENTANGLEMENT AND QUANTUM COMPUTATION: AN OVERVIEW
}

R. B. Perez

April 2000

\author{
Prepared by the \\ Oak Ridge National Laboratory \\ Oak Ridge, Tennessee 37831 \\ managed by \\ UT-Battelle, LLC \\ for \\ U.S. Department of Energy \\ under contract DE-AC05-00OR22725
}

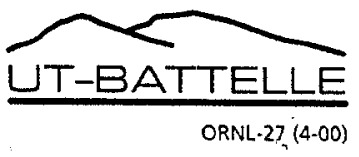





\title{
ENTANGLEMENT AND QUANTUM COMPUTATION:
} AN OVERVIEW

\author{
R. B. Perez $z^{*}$ \\ Instrumentation and Controls Division
}

April 2000

\author{
Prepared by \\ OAK RIDGE NATIONAL LABORATORY \\ Oak Ridge, Tennessee 37831-6004 \\ managed by \\ UT-BATTELLE, LLC \\ for the \\ U.S. DEPARTMENT OF ENERGY \\ under contract DE-AC05-00OR22725
}

* Signal and System Analysis, Inc. 
This page intentionally left blank. 


\section{CONTENTS}

ACKNOWLEDGMENT

ABSTRACT vii

1. INTRODUCTION

2. BRIEF REVIEW OF QUANTUM MECHANICS FUNDAMENTALS. 1

2.1 HILBERT SPACE

2.2 OPERATORS

2.2.1 Linear Operators...

2.2.2. Inverse Operators...........

2.2.3 Adjoint Operators

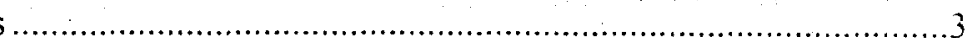

2.2.4 Hermitian (Self Adjoint) Operators................................................................

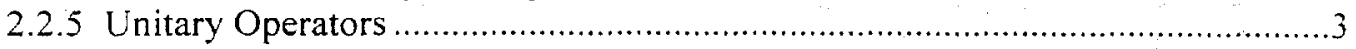

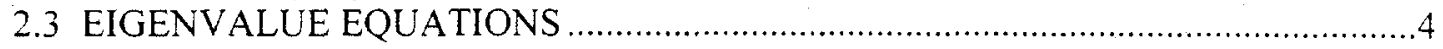

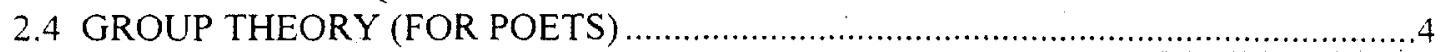

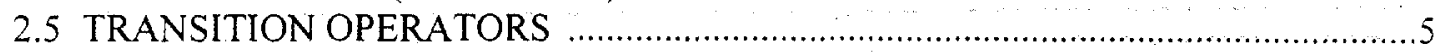

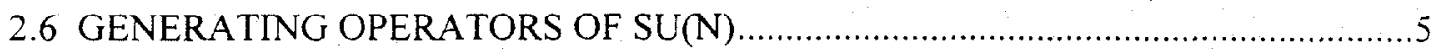

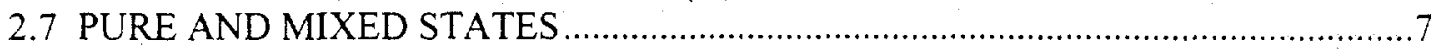

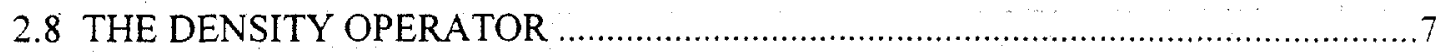

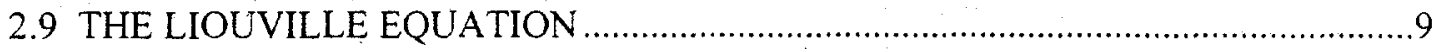

3. THE SUPERPOSITION PRINCIPLE AND SUPERSELECTION RULES $\ldots \ldots \ldots \ldots \ldots \ldots \ldots \ldots . \ldots \ldots$

3.1 SUPERSELECTION RULES.

4. COMPOSITE SYSTEMS: THE TENSOR PRODUCT OF HILBERT SPACES …............13

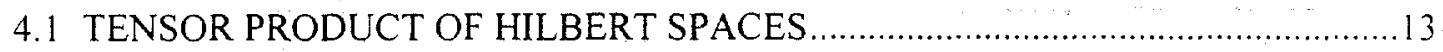

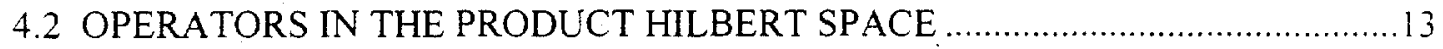

4.3 TENSOR PRODUCTS OF OPERATORS ........................................................ 14

4.4 THE DENSITY OPERATOR IN THE TENSOR PRODUCT SPACE:

ENTANGLEMENT

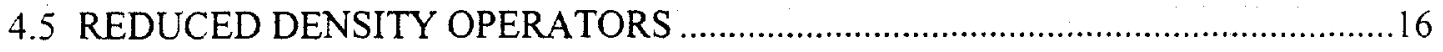

5. THE QUANTUM MECHANICAL DESCRIPTION OF MEASUREMENT ......................19

5.1 AXIOMATIC MEASUREMENT THEORY: THE PROJECTION POSTULATE

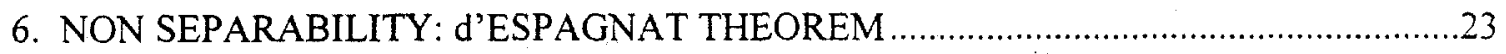

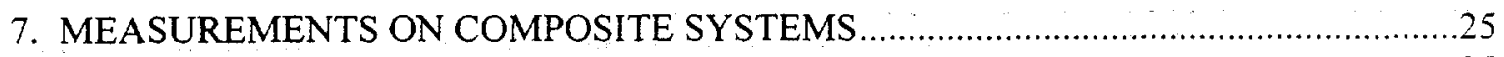

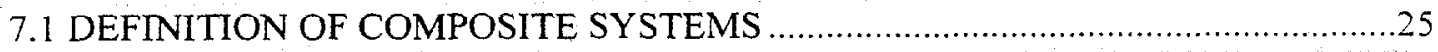

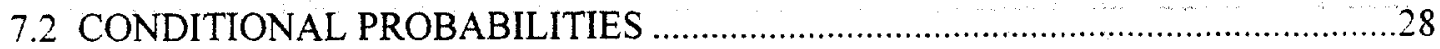

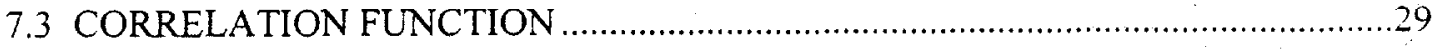

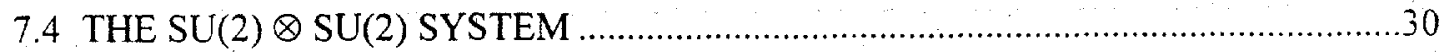

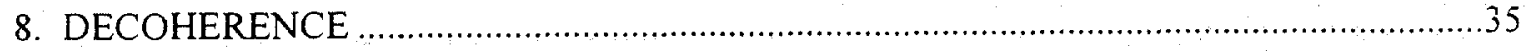




\section{CONTENTS (Cont'd.)}

9. THE AHARONOV-BOHM EFFECT

10. QUANTUM INTERFERENCE AND ENTANGLEMENT

OF MASSIVE PARTICLES

10.1 THE EINSTEIN-PODOLSKY-ROSEN (EPR) PARADOX

10.2 BOHR AND QUANTUM MECHANICS HIT BACK.

10.3 THE IRREDUCIBLE RANDOMNESS OF QUANTUM MECHANICS

10.4 ENTANGLEMENT AND BELL'S INEQUALITY

10.5 QUANTUM INTERFERENCE OF MASSIVE PARTICLES.

10.6 PARTICLE SELF-INTERFERENCF EXPERIMENTS.

10.7 QUANTUM INTERFERENCE EXPERIMENTS WITH CORRELATED MASSIVE PARTICLES.

10.8 NUCLEAR REACTIONS AS SOURCES OF CORRELATED

PARTICLES

11. QUANTUM COMPUTATION AND INFORMATION PROCESSING

11.1 QUBITS AND QUANTUM SOFTWARE

11.2 ENTANGLEMENT

11.3 DENSE CODING...

11.4 QUANTUM CRYPTOGRAPHY

11.5 QUANTUM TELEPORTATION

11.6 QUANTUM COMPUTER HARDWARE

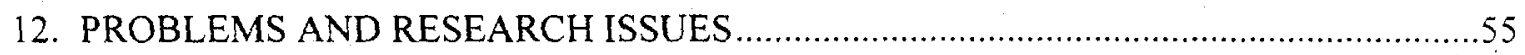

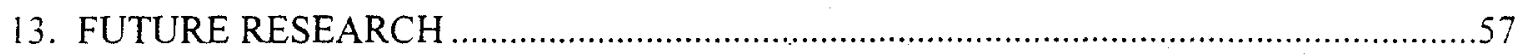

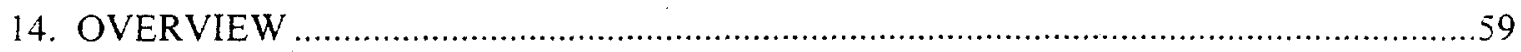

15. SHOULD ORNL BE INVOLVED IN QUANTUM INFORMATION PROCESSING RESEARCH?

16. REFLECTIONS ON QUANTUM MECHANICS

REFERENCES .65

APPENDIX A 69

APPENDIX B: PROJECTION POSTULATE .71

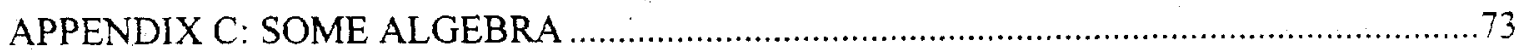

APPENDIX D: PROJECTION ALGORITHM FOR COMPOSITE SYSTEMS .......................75

APPENDIX E: CALCULATION OF $\mathrm{C}_{\mathrm{AA}}$ .77 
The authors are thankful to Dr. V. Protopopescu for many suggestions and criticisms as well as for his careful reading of the manuscript. We are indebted to Sherry Abercrombie for her patience and diligence in typing the many equations and text of this report. 
This page intentionally left blank. 
This report presents a selective compilation of basic facts from the ficlds of particle entanglement and quantum information processing prepared for those non-experts in these fields that may have interest in an area of physics showing counterintuitive. "spooky" (Einstein's words) behavior. In fact, quantum information processing could, in the near future, provide a new technology to sustain the benefits to the U.S. economy due to advanced computer technology. 
This page intentionally left blank. 
The quantum mechanical state of a system is said to be entangled when it arises from the superposition of states of identifiable correlated subsystems that can not be factorized. Entanglement is a pure quantum mechanical feature without a classical analogue.

In 1999 one of us, J. T. Mihalczo, suggested the use of nuclear reactions to produce entangled massive particles as an alternative to the already existing sources of entangled photons. Besides the High Flux Reactor, ORNL will be the site of the Spallation Source. both facilities providing the availability of the needed intense neutron sources able to induce a variety of nuclear reactions as a source of massive particles. It then appeared of some interest to know more about the fields of entanglement, quantum logic, and their possible future applications. Motivated by these ideas, it was decided to compile information on those fields, mostly to educate ourselves, in an area of physics showing counterintuitive "spooky" behavior. This report, like Gallia, is divided into three parts.

(a) Compilation of the quantum mechanical formalism needed to understand entanglement and non-locality in quantitative terms (the casual reader may skip most of this part). Most of this analysis has been based on the $S U(n)$ formalism as expounded in Reference 4. When needed, some of the mathematical steps have been worked out in various appendices. This part comprises Sections 2 up to 8.

(b) Sections 8 up to 10 deal with the subject of quantum interference and entanglement of massive particles. It also includes discussions on the Einstein-Podolski-Rosen paradox. Bell's inequalities, and the problem of measurement in quantum mechanics. A brief review of some interference measurements using massive particles is included as well.

(c) Due to the fact that the mathematical and physical background dealt with in parts (a) and (b) of the report is equally applicable to the fields of quantum logic and quantum information processing, the last part of this overview intends to provide a general description of the subjects of quantum cryptography, teleportation of quantum states. and quantum computers. The urgency for ORNL to participate in what is now a worldwide project on quantum logic is discussed in Section 15.

Clearly, this is not an in-depth review of the above-mentioned fields; it is rather an introduction for non-experts in quantum logic that, like ourselves, acquired some interested curiosity. As an aid, a relatively extensive list of references is provided. 


\section{SYMBOLS}

$\hat{1} \quad$ Unit Operator

$\hat{A}, \hat{B} \quad$ Operators

$T_{r} \quad$ Trace (sum over the diagonal elements)

$[\hat{\mathrm{A}}, \hat{\mathrm{B}}]$ Commutator, $(\hat{\mathrm{A}} \hat{\mathrm{B}}-\hat{\mathrm{B}} \hat{\mathrm{A}})$ 
This section contains a brief review of the quantum mechanical tools used in the following sections of this report. The fundamental ideas have been extracted from references 1.2. and 3. The development and application of the $S U(n)$ representation has been extracted entirely from Reference 4.

\subsection{HILBERT SPACE}

A Hilbert space is a complete normed space $\mathrm{H}$ in which the norm is given by a scalar product. A generic element of $\mathrm{H}, \mathrm{g}_{i}$, will be represented by the Dirac, ket. $\left|\mathrm{g}_{i}\right\rangle$. and its complex conjugated by the bra notations $<g_{i} \mid$. then the scalar product is written as $<g_{i} \mid g_{i}>$ $=\left\|g_{i}\right\|^{2}$.

\subsection{OPERATORS}

A rule $\hat{A}$ that transforms a vector, $g_{1} \in H_{1}$, to another vector, $g_{2} \in H_{2}$,

$$
g_{1}=\hat{A} g_{2} .
$$

\subsubsection{Linear Operators}

$$
\hat{\mathrm{A}}\left(\alpha_{1} \mathrm{~g}_{1}+\alpha_{2} \mathrm{~g}_{2}\right)=\alpha_{1} \hat{\mathrm{A}} \mathrm{g}_{1}+\alpha_{2} \hat{\mathrm{A}} \mathrm{g}_{2}
$$

\subsubsection{Inverse Operators}

Inverse operators, $\hat{\mathrm{A}}^{-1}$, defined by

$$
\hat{\mathrm{A}} \hat{\mathrm{A}}_{\mathrm{g}}^{-1}=\hat{\mathrm{A}}^{-1} \hat{\mathrm{A}} g=\hat{\mathrm{l}} \mathrm{g}=g \quad(\hat{\mathrm{l}}=\text { unit operator })
$$

\subsubsection{Adjoint Operators}

$$
\left\langle\hat{\mathrm{A}} \mathrm{g}_{2} \mid g_{1}\right\rangle=\left\langle g_{2} \mid \hat{\mathrm{A}}^{+} g_{1}\right\rangle
$$

\subsubsection{Hermitian (Self Adjoint) Operators}

$$
\hat{\mathrm{A}}^{+}=\hat{\mathrm{A}}
$$

\subsubsection{Unitary Operators}

$$
\hat{\mathrm{U}} \hat{\mathrm{U}}^{+}=\hat{1} ; \text { then }<\hat{\mathrm{U}} \mathrm{g}_{1}\left|\hat{\mathrm{U}} \mathrm{g}_{2}>=<g_{1}\right| g_{2}>
$$




\subsection{EIGENVALUE EQUATIONS}

The eigenvalue equation for the operator $\hat{\mathrm{A}}$ is

$$
\hat{\mathrm{A}}\left|\mathrm{g}_{1}>=a_{1}\right| g_{i}>
$$

where, $a_{\mathrm{i}}$, are the eigenvalues and $\mid g_{\mathrm{i}}>$ the eigenvectors. Orthonormality is represented by the inner product

$$
<g_{i} \mid g_{j}>=\delta_{i j}
$$

\subsection{GROUP THEORY (FOR POETS)}

A set $\mathrm{M}$ of elements is called a multiplicative group when:

(a) given a pair of elements, $A, B \in M$, the product $A B \in M(A B \neq B A)$;

(b) $(\mathrm{AB}) \mathrm{C}=\mathrm{A}(\mathrm{BC})$;

(c) There is a unit element, $1 \in \mathrm{M}$, such that $1 \mathrm{~A}=\mathrm{Al}=\mathrm{A}$;

(d) Given $A \in M$, there is an inverse element $A^{-1} \in M$ such that $A^{-1}=A^{-1} A=1$.

In the case that condition (d) is not fulfilled, the membership is called a semigroup. The group of operators, $U$, (with elements $U_{i j}$ ) that transforms n-dimensional vectors, $\overrightarrow{\mathrm{v}}=\left(\mathrm{v}_{1}, \mathrm{v}_{2}, \ldots \mathrm{v}_{n}\right)$, according to

$$
\mathrm{v}^{\prime}{ }_{i}=\sum_{j=1}^{n} \mathrm{U}_{i j} \mathrm{v}_{j}
$$

is called the unitary group, $U(n)$, if

$$
\mathrm{UU}^{+}=\mathrm{U}^{+} \mathrm{U}=1 \text { (i.e. } \sum_{\mathrm{j}=1}^{\mathrm{n}} \mathrm{U}_{\mathrm{ij}} \mathrm{U}_{k j}^{*}=\sum_{j} \mathrm{U}_{j i}^{*} \mathrm{U}_{j k}=\delta_{i k} \text { ). }
$$

Ilence, $|\operatorname{det}(\mathrm{U})|=1$; if $\operatorname{det}(\mathrm{U})=1$, the unitary group is called a special unitary group, $\mathrm{SU}(\mathrm{n})$. 


\subsection{TRANSITION OPERATORS}

Given the eigenvectors $|\mathrm{k}\rangle, \mid \ell>$, the transition operator $\hat{\mathrm{P}}_{k l}$ is defined as

$$
\hat{\mathrm{P}}_{k \ell}=\left|k>\int \mathrm{d} \underline{\mathrm{r}}<\ell\right| \quad(\text { or simply }|\mathrm{k}><\ell|, \text { reminding the integration step) }
$$

To see how it works, consider the ket, $\mid \Psi>$, expanded in terms of eigenvectors $\mid k>$. i.e.,

$$
\left|\Psi>=\sum_{k^{\prime}} a_{k^{\prime}}\right| k^{\prime}>
$$

apply now the transition operator $\hat{\mathrm{P}}_{\mathrm{k} \mathrm{k}}$ :

$$
\hat{P}_{k k}|\Psi\rangle=\mid k>\sum_{k^{\prime}} \int \mathrm{d} \underline{r}\left\langle k\left|a_{k^{\prime}}\right| k^{\prime}>=\left|k>\sum_{k^{\prime}} a^{\prime}{ }_{k}<k\right| k^{\prime}>=\left|k>\sum_{k^{\prime}} a_{k}^{\prime} \delta^{\prime}{k^{\prime} k}^{\prime}=a_{k}\right| k>\right.
$$

the result is to "project out" the k-th component of $|\psi\rangle$, so that $\hat{\mathrm{P}}_{\mathrm{kk}}$ can be termed a projection operator. Important properties of these operators ${ }^{4}$ are:

$$
\begin{aligned}
& \sum_{k p} \hat{\mathrm{P}}_{k \ell}=\hat{\mathrm{l}} ; \sum_{k} \hat{\mathrm{P}}_{k k}=\hat{\mathrm{l}} \\
& \hat{\mathrm{P}}_{k \ell} \hat{\mathrm{P}}_{m n}=\hat{\mathrm{P}}_{k n} \delta_{\ell m}
\end{aligned}
$$

with the commutator relation

$$
\left[\hat{\mathrm{P}}_{k \ell}, \hat{\mathrm{P}}_{m n}\right]=\hat{\mathrm{P}}_{k !} \delta_{l m}-\hat{\mathrm{P}}_{m \ell} \delta_{n k} .
$$

The following trace relations apply

$$
\mathrm{T}_{r}\left\{\hat{\mathrm{P}}_{k \ell}\right\}=\delta_{k \ell} ; \quad \operatorname{Tr}\left\{\hat{\mathrm{P}}_{k \ell} \hat{\mathrm{P}}_{m n}\right\}=\delta_{\ell m} \delta_{k n}
$$

\subsection{GENERATING OPERATORS OF SU(n)}

The elements of a group can be constructed on the basis of a set of quantities called generating operators (or group generators). All $(n x n)$ unitary matrices with $\operatorname{det}(U(n))=1$, define the subgroup $S U(n)$. The elements $U_{i j}$ can be represented in terms of a Hermitian matrix, A, by:

$$
\mathrm{U}_{n m}=\exp \left\{\mathrm{i} \mathrm{A}_{n m}\right\} ; \mathrm{A}_{n m}=\mathrm{A}_{m n}^{*}
$$


In view of the condition, $\operatorname{det} \mathrm{U}(\mathrm{n})=1$, and the hermiticity of $\underline{\mathrm{A}}$, there are $\mathrm{s}=\mathrm{n}^{2}-1$ independent generating operators, $\lambda,(j=1,2 \ldots s)$ and the rank of the group, $r$, is equal to $(n-1)$.

The generating operators, $\hat{\lambda}_{j}(j=1,2, \ldots s)$ are Hermitian operators defined by the relations

$$
\left[\hat{\lambda}_{i}, \hat{\lambda}_{i}\right]=2 i \sum_{k=1}^{s} \mathrm{f}_{i j k} \hat{\lambda}_{k}
$$

The quantities $\mathrm{f}_{\mathrm{ijk}}$, are the so-called structure constants. The $\lambda$ - operators obey the trace relations:

$$
\operatorname{T}_{r}\left\{\hat{\lambda}_{j}\right\}=0 ; \operatorname{Tr}\left\{\hat{\lambda}_{1} \hat{\lambda}_{k}\right\}=2 \delta_{j k} .
$$

The $S U(n)$ generators are expressed in terms of transition operators:

$$
\overrightarrow{\hat{\lambda}}=\left\{\hat{\mathrm{u}}_{12}, \hat{\mathrm{u}}_{13}, \hat{\mathrm{u}}_{23}, \ldots \hat{\mathrm{v}}_{12}, \hat{\mathrm{v}}_{13}, \hat{\mathrm{v}}_{23}, \ldots \hat{\mathrm{w}}_{1}, \hat{\mathrm{w}}_{2}, \ldots \hat{\mathrm{w}}_{n-1}\right\} ;
$$

with

$$
\begin{aligned}
& \hat{\mathrm{u}}_{j k}=\hat{\mathrm{P}}_{j k}+\hat{\mathrm{P}}_{k j} ; \hat{\mathrm{v}}_{j k}=\mathrm{i}\left(\hat{\mathrm{P}}_{\mathrm{jk}}-\hat{\mathrm{P}}_{k j}\right) \\
& \hat{\mathrm{w}}_{\ell}=-\sqrt{\frac{2}{\ell(\ell+1)}}\left(\hat{\mathrm{P}}_{11}+\ldots \hat{\mathrm{P}}_{\ell \ell}-\ell \hat{\mathrm{P}}_{\ell+1, \ell+1}\right) \\
& (1 \leq \mathrm{j}<\mathrm{k} \leq \mathrm{n} ; 1 \leq \ell \leq \mathrm{n}-1) .
\end{aligned}
$$

A given Hermitian operator, $\hat{A}$, acting on an $n$-dimensional Hilbert space, can be represented in terms of the $\mathrm{SU}(\mathrm{n})$ generators:

$$
\hat{\mathrm{A}}=\frac{1}{\mathrm{n}} \mathrm{A}_{\nu} \hat{1}+\frac{1}{2} \sum_{j=1}^{s} \mathrm{~A}_{j} \hat{\lambda}_{j} ; \mathrm{A}_{o}=\mathrm{T}_{r}\{\hat{\mathrm{A}}\} ; \mathrm{A}_{\mathrm{j}}=\mathrm{T}_{r}\left\{\hat{\mathrm{A}} \hat{\lambda}_{j}\right\}
$$

An example is worked out in Appendix A.

In particular, because the $\hat{\mathrm{w}}_{\ell}$ operators together with the unit operator form a complete set of commuting operators, one can rewrite (2.26) in the form

$$
\hat{\mathrm{A}}=\frac{1}{\mathrm{n}} \mathrm{A}_{o} \hat{\mathrm{l}}+\frac{1}{2} \sum_{\ell+1}^{n-1} \mathrm{~A}_{\ell} \hat{\mathrm{w}}_{\ell} ; \mathrm{A}_{o}=\mathrm{T}_{r}\{\hat{\mathrm{A}}\} ; \mathrm{A}_{\ell}=\mathrm{T}_{r}\left\{\hat{\mathrm{A}} \mathrm{w}_{\ell}\right\}
$$


The eigenvalue equations associated with the $\hat{\mathrm{w}}_{\ell}$ operators are

$$
\hat{\mathrm{w}}_{\ell}\left|v>=\hat{\mathrm{w}}_{\ell}^{(v)}\right| v>\quad(1 \leq v \leq \mathrm{n}, 1 \leq \ell \leq \mathrm{n}-1)
$$

with the eigenvalues

$$
\begin{aligned}
& \mathrm{w}_{\ell}^{(v)}=-\left[\frac{2}{\ell(\ell+1)}\right]^{\frac{1}{2}} ; 1 \leq v \leq \ell \\
& \mathrm{w}_{\ell}^{(\ell+1)}=\ell\left[\frac{2}{\ell(\ell+1)}\right]^{\frac{1}{2}} ; \quad v=\ell+1 \\
& \mathrm{w}_{\ell}^{(\nu)}=0 ; \quad \ell+1<v \leq \mathrm{n} .
\end{aligned}
$$

\subsection{PURE AND MIXED STATES}

When a quantum system can be described by a single state vector, $\mid \psi>$, given in terms of an orthonormal basis by

$$
\left|\Psi>=\sum_{n} c_{n}\right| n>
$$

the corresponding state is called a "pure" state.

In the presence of fluctuations, due for instance to interaction with the environment, the system may find itself in various state $\mid \psi_{v}>$ occurring with probability, $p_{v}$, in this instance, the state of the system is called a mixed state.

\subsection{THE DENSITY OPERATOR}

For a pure state the density operator is defined as

$$
\hat{\rho}=|\Psi><\Psi|
$$

It coincides with the projection operator on the state $|\Psi\rangle$.

Its matrix elements, $\rho_{\mathrm{ij}}$, define the density matrix. From (2.33) and (2.32)

and

$$
\rho_{j k}=\langle\mathrm{j}|\hat{\rho}| \mathrm{k}\rangle=\langle\mathrm{j} \mid \Psi\rangle\langle\Psi \mid \mathrm{k}\rangle=\mathrm{c}_{j} \dot{\mathrm{c}_{k}}
$$




$$
\hat{\rho}=\sum_{j k} c_{j} \dot{c}_{k}|j><\mathrm{k}|
$$

The expectation value of an operator, $\hat{\mathrm{A}}$, is defined as

$$
\langle\hat{\mathrm{A}}\rangle=\langle\Psi|\hat{\mathrm{A}}| \Psi\rangle=\sum_{\mathrm{jk}}\left\langle\mathrm{j}\left|\mathrm{c}_{j}^{*} \hat{\mathrm{A}} \mathrm{c}_{\mathrm{k}}\right| \mathrm{k}\right\rangle=\sum_{\mathrm{jk}} \mathrm{c}_{\mathrm{j}}^{*} c_{k}\langle\mathrm{j}|\mathrm{A}| \mathrm{k}\rangle
$$

Insert now (2.7) and use (2.8) to get

$$
<\hat{\mathrm{A}}>=\sum_{j k} \dot{\mathrm{c}_{j}} \mathrm{c}_{k} \mathrm{a}_{k}<\mathrm{j} \mid \mathrm{k}>=\sum_{\mathrm{j}} \mathrm{c}_{j} \dot{\mathrm{c}_{j}} \mathrm{a}_{j}
$$

It is convenient to express expectation values in terms of the density operator. Consider the quantity

$$
\left.\mathrm{Q}=\mathrm{T}_{\mathrm{r}}\{\hat{\mathrm{A}} \hat{\rho}\}=\mathrm{T}_{r}\left\{\sum_{j k} \mathrm{c}_{j} \mathrm{c}_{k}^{*}<\mathrm{j}|\hat{\mathrm{A}}| \mathrm{k}>\right\}=\sum_{j} \mathrm{c}_{j} \mathrm{c}_{j}^{*}<\mathrm{j}|\hat{\mathrm{A}}| \mathrm{j}\right\rangle=\sum_{j} \mathrm{c}_{j} \mathrm{c}^{*} \mathrm{a}_{j}=\langle\hat{\mathrm{A}}>
$$

hence we obtain the useful result

$$
<\hat{\mathrm{A}}>=\mathrm{T},\{\hat{\rho} \hat{\mathrm{A}}\} \text {. }
$$

For a mixed state, the density operator is

$$
\hat{\rho}=\sum_{v} \mathrm{p}_{v}\left|\Psi_{v}><\Psi_{v}\right|
$$

and the expectation values are now

$$
<\hat{\mathrm{A}}>=\sum_{v} \mathrm{p}_{v}\left\langle\Psi_{v}|\hat{\mathrm{A}}| \Psi_{v}\right\rangle
$$

The density operator satisfies the relation

$$
T_{r}\{\hat{\rho}\}=\hat{1}
$$

For later work it becomes convenient to write the density operator in terms of the SU(n) generators, $\lambda_{j}$. We use equations (2.26) and (2.41) to get

$$
\hat{\rho}=\frac{1}{\mathrm{n}} \hat{1}+\frac{1}{2} \sum_{j=1}^{s} \lambda_{j} \hat{\lambda}_{j} ; \lambda_{j}=<\hat{\lambda}_{j}>=\operatorname{Tr}\left\{\hat{\rho} \hat{\lambda}_{j}\right\}
$$


The real valued expectation values, $\lambda_{j}$, form the so-called coherence vector $\lambda_{1}$ $(\lambda, j=1 \ldots s)$.

\subsection{THE LIOUVILLE EQUATION}

Upon operating on (2.33) with, i $\hbar \frac{\partial}{\partial t}$, and after use is made of the Schrödinger equation: $i \hbar \frac{\partial}{\partial t}|\Psi>=\hat{H}| \Psi>$, satisfied by the state vector $\mid \Psi>$ and its complex conjugate one obtains the quantum mechanical version of the Liouville equation

$$
i \hbar \frac{\partial}{\partial t} \hat{\rho}=\hat{\mathrm{H}} \hat{\rho}-\hat{\rho} \hat{\mathrm{H}} \equiv[\hat{\mathrm{H}}, \hat{\rho}]
$$

where $\hat{\mathrm{H}}$ is the Hamiltonian operator.

For an isolated physical system, the Liouville equation is the mathematical representation of the quantum mechanical principle of the deterministic evolution of the system; i.e., if at $t=t_{0}$, the density operator is $\rho\left(t_{0}\right)$ at a later time, one has $\rho(t)$ given by

$$
\hat{\rho}(t)=\hat{\mathrm{U}}\left(\mathrm{t}-\mathrm{t}_{0}\right) \hat{\rho}\left(\mathrm{t}_{0}\right) \hat{\mathrm{U}}^{-1}\left(\mathrm{t}-\mathrm{t}_{o}\right)
$$

where $U\left(t-t_{0}\right)$ is a unitary operator. Note that the process described by Eq. (2.44) is reversible and as such it does not change the system's entropy. 
This page intentionally left blank. 
Dirac postulated the superposition principle as the fundamental principle of quantum theory. We follow Dirac's line of thought so brilliantly expressed in his classic text. "The Principles of Quantum Mechanics." Dirac introduces the superposition of states idea using the example of a beam of light passing through a polarizing crystal. Consider the case of a single obliquely polarized photon at an angle, $\alpha$, with respect to the crystal optic axis. After several measurements, one will find the whole photon on the backside in a fraction, $\sin ^{2} \alpha$, of the total number of trials. "The result of an experiment is not determined, as it would be according to classical ideas, by the conditions under the control of the experimenter. The most than can be predicted is a set of possible results, with a probability of occurrence for each." The quantum mechanical explanation, secundum Dirac, considers that a photon polarized obliquely to the optic axis is partly in the state of polarization parallel to the axis and partly in the state of polarization perpendicular to the axis. Given the quantum systems, $A$ and $B$, represented by the kets, $|A\rangle,|B\rangle$, respectively, if an state results from the superposition of the $A$ and $B$ states, the associated ket vector, $|R\rangle$, can be expressed as a linear combination; i.e.,

$$
\left|\mathrm{R}>=\mathrm{C}_{1}\right| \mathrm{A}>+\left.\mathrm{C}_{2}|\mathrm{~B}>;| \mathrm{C}_{1}\right|^{2}+\left|\mathrm{C}_{2}\right|^{2}=1
$$

where, $\mathrm{C}_{1}$ and $\mathrm{C}_{2}$, are complex numbers.

Dirac completes the mathematical formulation contained in Eq. (3.1) by introducing a further assumption, "The superposition of an state with itself cannot form any new state," i.e.,

$$
C_{1}\left|A>+C_{2}\right| A>=\left(C_{1}+C_{2}\right) \mid A>
$$

It follows from the statement involved in (3.3) that $\left(C_{1}+C_{2}\right) \mid A>$ must be the same state that the one represented by $|A\rangle$. Thus, a state is specified by the direction of the ket vector in $\mathcal{H}$-space, the length of the vector being irrelevant. These properties show the fundamental difference between the superposition of the quantum theory and the kind of superposition process in classical theory. In the later context when one superposes one state with itself, for instance a vibrating string, one obtains a different state with a different oscillatory strength. Note that a superposition of states is "its own" state, different from the set of its components. A spectacular success of the superposition principle was the prediction of superpositions of $\mathrm{K}$-mesons and their antiparticles as forming new and different particles. ${ }^{6}$ The superposition principle when combined with quantization gives rise to "entanglement".

\subsection{SUPERSELECTION RULES}

There are, however, state superpositions that are not conceivable seen in Nature. Some physicists, among them Wigner, ${ }^{3}$ have postulated "superselection rules" that restrict the superposition principle. One of the most important excludes superposition of states with integer and half spins or having different electrical charges. 
This page intentionally left blank. 


\section{COMPOSITE SYSTEMS: THE TENSOR PRODUCT OF HILBERT SPACES}

Starting from two independent systems, each one described by its own Hilbert space, an appropriate mathematical scheme to describe composite states is to construct a single larger Hilbert space as a product of the two. ${ }^{3,4}$

\subsection{TENSOR PRODUCT OF HILBERT SPACES}

The composite system of two subsystems defined in $\mathrm{H}(1), \mathrm{H}(2)$, exists in the product Hilbert space

$$
H(1,2)=H(1) \otimes H(2)
$$

Denoting by $\mid \ell(1)>$ and $\mid \ell(2)>$ the state vectors in systems (1) and (2), one has the completeness relations

$$
\hat{1}(v)=\sum_{\ell}|\ell(v)><\ell(v)| \quad(v=1,2)
$$

The state vectors $\mid \ell(v)>$, span the H-spaces, $H(v)(v=1,2)$, with dimensions

$$
\operatorname{dim} H(v)=\mathrm{n}_{v}
$$

The state vector spanning the composite system, is the tensor product

$$
|\ell(1) \mathrm{m}(2)>=| \ell(1)>\otimes|\mathrm{m}(2)>=| \mathrm{m}(2)>\otimes \mid \ell(1)>
$$

Completeness for the product $\mathrm{H}$-space is

$$
\hat{1}=\hat{1}(1) \otimes \hat{1}(2)=\sum_{\ell, m}|\ell(1) m(2)><\ell(1) \mathrm{m}(2)|
$$

The product state vectors satisfy the orthonormality relation

$$
<\ell(1) \mathrm{m}(2) \mid \ell^{\prime}(1) \mathrm{m}^{\prime}(2)>=\delta_{\rho f^{\prime}} \delta_{m m^{\prime}}
$$

\subsection{OPERATORS IN THE PRODUCT HILBERT SPACE}

Let the operator $\hat{A}(1)$ be in $H(1)$ and the operator $\hat{B}(2)$ in $H(2)$, with eigenvalue equations and unit eigenvalues

$$
\left.\hat{\mathrm{A}}(1)|\ell(1)>=| \ell^{\prime}(1)>; \hat{\mathrm{B}}(2)|\mathrm{m}(2)>=| \mathrm{m}^{\prime}(2)\right\rangle
$$


then in the product Hilbert space, $\mathrm{H}$, these operators are

$$
\hat{\mathrm{A}}=\hat{\mathrm{A}}(1) \otimes \hat{\mathrm{I}}(2) ; \hat{\mathrm{B}}=\hat{\mathrm{l}}(1) \otimes \hat{\mathrm{B}}(2)
$$

The eigenvalue equations (4.7) become in the product space

$$
\hat{\mathrm{A}}|\ell(1) \mathrm{m}(2)>=| \ell^{\prime}(1) \mathrm{m}(2)>; \hat{\mathrm{B}}|\ell(1) \mathrm{m}(2)>=| \ell(1) \mathrm{m}^{\prime}(2)>
$$

The matrix elements of the $\hat{A}$ and $\hat{B}$ operators are given by

$$
\begin{aligned}
& <\ell(1) \mathrm{m}(2)|\hat{\mathrm{A}}| \ell^{\prime}(1) \mathrm{m}^{\prime}(2)>=\mathrm{A}_{\varphi \mu^{\prime}}(1) \delta_{m m^{\prime}} \\
& <\ell(1) \mathrm{m}(2)|\hat{\mathrm{B}}| \ell^{\prime}(1) \mathrm{m}^{\prime}(2)>=\mathrm{B}_{m m^{\prime}}(2) \delta_{\ell \ell^{\prime}}
\end{aligned}
$$

where, $\mathrm{A}(1)_{\epsilon \ell^{\prime}}, \mathrm{B}(2)_{m m^{\prime}}$, are matrix elements in the subspaces $\mathrm{H}_{1}, \mathrm{H}_{2}$.

From (4.9) and (4.10) important trace relations arise

$$
\begin{aligned}
& <\ell(1)\left|\mathrm{T}_{r 2}\{\hat{\mathrm{A}}\}\right| \ell^{\prime}(1)>=\mathrm{A}_{r \prime^{\prime}}(1) \mathrm{n}_{2} \\
& <\mathrm{m}(2)\left|\mathrm{T}_{\mathrm{r} 1}\{\hat{\mathrm{B}}\}\right| \mathrm{m}^{\prime}(2)>=\mathrm{n}_{1} \mathrm{~B}_{m n^{\prime}}(2)
\end{aligned}
$$

where, $T_{r_{i j}}(i=1,2)$ means that the trace operation is performed on the $\mathrm{i}^{\text {th }}$ subspace only, and $\mathrm{n}_{1}$ and $\mathrm{n}_{2}$ are the dimensions of the Hilbert subspaces. Also

$$
\begin{aligned}
& \mathrm{T}_{r}\{\hat{\mathrm{A}}\}=\sum_{\ell} \mathrm{A}(1)_{\ell \ell} \mathrm{n}_{2}=\mathrm{T}_{r 1}\{\hat{\mathrm{A}}(1)\} \mathrm{n}_{2} \\
& \mathrm{~T}_{r}\{\hat{\mathrm{B}}\}=\sum_{m} \mathrm{n}_{1} \mathrm{~B}_{m m}(2)=\mathrm{n}_{1} \mathrm{~T}_{r 2}\{\hat{\mathrm{B}}(2)\} .
\end{aligned}
$$

\subsection{TENSOR PRODUCTS OF OPERATORS}

Starting from subsystem operators, one can form new tensor product of operators

$$
\hat{\mathrm{A}}(1,2)=\hat{\mathrm{A}}(1) \otimes \hat{\mathrm{B}}(2)=\hat{\mathrm{B}}(2) \otimes \hat{\mathrm{A}}(1) .
$$

The following rules apply

$$
[\hat{\mathrm{A}}(1) \otimes \hat{\mathrm{B}}(2)][\hat{\mathrm{C}}(1) \otimes \hat{\mathrm{D}}(2)]=\hat{\mathrm{A}}(1) \hat{\mathrm{C}}(1) \otimes \hat{\mathrm{B}}(2) \hat{\mathrm{C}}(2)
$$




$$
[\hat{\mathrm{A}}(1) \otimes \hat{\mathrm{B}}(2), \hat{\mathrm{C}}(1) \otimes \hat{\mathrm{D}}(2)]=\hat{\mathrm{A}} \hat{\mathrm{C}} \otimes \hat{\mathrm{B}} \hat{\mathrm{D}}-\hat{\mathrm{C}} \hat{\mathrm{A}} \otimes \hat{\mathrm{D}} \hat{\mathrm{B}}
$$

The matrix elements associated with the product are given by the product of the matrix elements in each subspace:

$$
<\ell(1) m(2)|\hat{\mathrm{A}}(1) \otimes \hat{\mathrm{B}}(2)| \ell^{\prime}(1) \mathrm{m}^{\prime}(1)>=\mathrm{A}_{\mu^{\prime}}(1) \mathrm{B}_{m m^{\prime}}(2) .
$$

From (4.19) it follows that

$$
\mathrm{T}_{r}\{\hat{\mathrm{A}}(1) \otimes \hat{\mathrm{B}}(2)\}=\sum_{\gamma_{1, \mathrm{~m}}} \mathrm{~A}(1)_{n} \mathrm{~B}(2)_{\min }=\mathrm{T}_{\mathrm{r}_{1}}\{\hat{\mathrm{A}}(1)\} \mathrm{T}_{\mathrm{r}_{2}}\{\hat{\mathrm{B}}(2)\}
$$

From the above developments, one derives two useful relations for the direct products of $\mathrm{SU}(\mathrm{n})$ generators:

(a) From (4.20) and (2.22)

$$
\mathrm{T}_{r}\left\{\hat{\lambda}_{j}(1) \otimes \hat{\lambda}_{k}(2)\right\}=0
$$

(b) From (4.17) and (2.22)

$$
\begin{aligned}
& \mathrm{T}_{r}\left\{\left[\hat{\lambda}_{j}(1) \otimes \hat{\lambda}_{k}(2)\right]\left[\hat{\lambda}_{l}(1) \otimes \lambda_{m}(2)\right\}\right. \\
& =\mathrm{T}_{1}\left\{\hat{\lambda}_{j}(1) \hat{\lambda}_{\ell}(1)\right\} \mathrm{T}_{r_{2}}\left\{\hat{\lambda}_{k}(2) \hat{\lambda}_{m}(2)\right\}=4 \delta_{j l} \delta_{k m} .
\end{aligned}
$$

\subsection{THE DENSITY OPERATOR IN THE TENSOR PRODUCT SPACE: ENT ANGLEMENT}

Based on the algebra for the tensor product of operators in the previous sections and on equation (2.42), he density operator for a composite system is written in terms of SU(n), generators, as

$$
\begin{aligned}
\hat{\rho}(1,2)=\frac{1}{\mathrm{n}_{1} \mathrm{n}_{2}} \hat{1}(1) \otimes \hat{1}(2) & +\frac{1}{2 \mathrm{n}_{1}} \sum_{j=1}^{s 1} \lambda_{j}(1)\left[\hat{\lambda}_{j}(1) \otimes \hat{1}(2)\right] \\
& +\frac{1}{2 \mathrm{n}_{2}} \sum_{k=1}^{s 2} \lambda_{k}(2)\left[\hat{1}(1) \otimes \hat{\lambda}_{k}(2)\right] \\
& +\frac{1}{4} \sum_{j, k}^{11.52} \mathrm{~K}_{j k}(1,2)\left[\hat{\lambda}_{j}(1) \otimes \hat{\lambda}_{k}(2)\right]
\end{aligned}
$$

where $\lambda_{j}(1)\left(\mathrm{j}=1,2, \ldots \mathrm{n}_{1}\right) ; \lambda_{j}(2)\left(\mathrm{k}=1,2, \ldots \mathrm{n}_{2}\right)$ are $\mathrm{SU}(\mathrm{n})$ generators for systems $(1)$ and (2). The quantities, $\lambda_{j}(1), \lambda_{k}(2)$ are the expectation values 


$$
\begin{aligned}
& \lambda_{,}(1)=\operatorname{T}_{r}\left\{\hat{\rho}\left[\hat{\lambda}_{j}(1) \otimes \hat{l}(2)\right]\right\} \\
& \lambda_{k}(2)=T_{r}\left\{\hat{\rho}\left[\hat{l}(1) \otimes \hat{\lambda}_{k}(2)\right]\right\}
\end{aligned}
$$

and the real valued quantities $\mathrm{K}_{\mathrm{jk}}(1,2)$ are the components of the correlation tensor:

$$
\mathrm{K}_{j k}(1,2)=<\hat{\lambda}_{j}(1) \hat{\lambda}_{k}(2)>=\mathrm{T}_{r}\left\{\hat{\rho}\left[\hat{\lambda}_{j}(1) \otimes \hat{\lambda}_{k}(2)\right]\right\} .
$$

One can also introduce the "covariance" (correlation) matrix

$$
\mathrm{M}_{j k}=\mathrm{K}_{j k}-\lambda,(1) \lambda_{k}(2)
$$

On the basis of (4.27) a composite system will be entangled whenever $\mathrm{M}_{\mathrm{jk}} \neq 0$. Note that for a product state (total wave function equal to the product of wave functions) $\mathrm{M}_{\mathrm{jk}}$ becomes zero.

\subsection{REDUCED DENSITY OPERATORS}

In this section (following Reference 4) we define the concept of reduced density operators. $R(1), R(2)$, allowing for the calculation of expectation values for the subsystem $H(1)$ and $\mathrm{H}(2)$.

Recall that we are now in the composite Hilbert space where it exists a density operator

$$
\hat{\rho}(1,2)=\sum_{\ell j m k} \mathrm{P}_{\ell j m k}|\ell(1) \mathrm{m}(2)><\mathrm{j}(1) \mathrm{k}(2)|
$$

with the matrix element

$$
\rho_{\ell j m k}=<\ell(1) \mathrm{m}(2)|\hat{\rho}| \mathrm{j}(1) \mathrm{k}(2)>.
$$

One desires to calculate the expectation value of the operator $\hat{A}(1)$ in the subsystems $\mathrm{H}(1)$. We use (2.38) and (4.8), i.e.,

$$
<\hat{\mathrm{A}}>=\mathrm{T}_{r}\{\hat{\rho} \mid \hat{\mathrm{A}}(1) \otimes \hat{\mathrm{I}}(2)\}
$$

that in view of (4.28) and (4.29) can be rewritten as

$$
\langle A\rangle=\sum_{i j m k}[\langle\ell(1) m(2)|\hat{\rho}| j(1) k(2)>]<j(1) k(2)|\hat{A}(1) \otimes \hat{i}(2)| \ell(1) m(2)\rangle
$$


Use now (4.19) with $\hat{\mathrm{B}}(2)=\hat{1}(2)$ to get

$$
<\hat{\mathrm{A}}(1)>=\sum_{i j \mathrm{~m}}<\ell(1) \mathrm{m}(2)|\hat{\rho}| j(1) \mathrm{m}(2)>\mathrm{A} j \ell
$$

Define the reduced density matrix

$$
\mathrm{R}_{\ell,}(1)=\sum_{m}<\ell(1) \mathrm{m}(2)|\hat{\rho}| \mathrm{j}(1) \mathrm{m}(2)>
$$

so that now

$$
<\hat{\mathrm{A}}(1)>=\sum_{i j} \mathrm{R}_{\gamma^{\prime}}(1) \mathrm{A}_{\gamma^{\ell}}
$$

Observe that corresponding to the reduced density matrix elements, $R_{i j}(1)$, one must have a reduced density operator, $\hat{R}(1)$ such that

$$
\mathrm{R}_{\ell}(1)=\langle\ell(1)|\hat{\mathrm{R}}(1)| \mathrm{j}(1)>
$$

hence comparing (4.32) and (4.34)

$$
\hat{\mathrm{R}}(1)=\sum_{\mathrm{m}}<\mathrm{m}(2)|\hat{\rho}| \mathrm{m}(2)>=\mathrm{T}_{\mathrm{r}_{2}}\{\hat{\rho}\}
$$

Similarly, one obtains

$$
\hat{\mathrm{R}}(2)=\mathrm{T}_{\mathrm{r}_{1}}\{\hat{\rho}\} \text {. }
$$

One concludes then that to obtain information on the subsystem (1) one must trace out the subsystem (2) and vice versa. 
This page intentionally left blank. 


\section{THE QUANTUM MECHANICAL DESCRIPTION OF MEASUREMENT}

We follow the general lines of thought pursued by Wigner ${ }^{7}$ and London and Bauer ${ }^{8}$ in the analysis of the quantum mechanical process of measurement. We assume that in the remote past, the "object" an the measuring apparatus existed in two separate Hilbert spaces. $\mathrm{H}(1)$ and $\mathrm{H}(2)$, respectively. The property of the object to be measured is an observable associated to a Hermitian operator, $\hat{\mathrm{L}}$, characterized by an eigenset $\left|\ell_{k}(1)\right\rangle$ corresponding to the eigenvalues $a_{k}$. The measuring apparatus is in turn described by another Hermitian operator, $\hat{\mathrm{G}}$, describing the coordinates of the "pointers" of the measuring device. Associated to this operator there is a set of eigenfunctions $m_{k}(2)$ and eigenvalues, $g_{k}$, corresponding to "pointers" locations. In $\mathrm{H}(1)$ the object is in the pure state

$$
\left|\ell(1)>=\sum_{k} \alpha_{k}\right| \ell_{k}(1)>
$$

whereas the measuring apparatus is assumed to be on its "zero" state, $\mathrm{m}_{0}(2)$.

The act of measurement is formulated mathematically by the construction of the product Hilbert space, $\mathrm{H}=\mathrm{H}(1) \otimes \mathrm{H}(2)$. Then according to (4.4) the state vector spanning, $\mathrm{H}$, is the product state:

$$
\left|\ell(1) \mathrm{m}_{\iota}(2)>=\right| \mathrm{m}_{\iota}(2)>\sum_{k} \alpha_{k} \mid \ell_{k}(1)>
$$

The ket, (5.2) is the solution of a wave equation controlled by a Hamiltonian split in two parts, each one of them depending only on a set of coordinates (either (1) or (2)). After the introduction of an interaction object-apparatus, that depends on both sets of coordinates the resulting state, $\mid \Psi(1,2)>$ can be expressed in terms of direct products $\mid \ell(1) m(2)>$, because they constitute a set of complete orthogonal kets [see equations ( 4.5 and 4.6$)$ ], i.e.,

$$
|\Psi(1,2)\rangle=\sum_{k k^{\prime}} \alpha_{k k^{\prime}}\left|\ell_{k}(1) \mathrm{m}_{k^{\prime}}(2)\right\rangle
$$

The results (5.3) to provide a measurement must correlate the pointer positions, $g_{v}$, with the quantities to be measured, $a_{v}$, in a one-to-one manner. It is then that one can inscribe directly onto the $g_{v}$ scale the corresponding values, $a_{v}$. The present form (5.3) does not satisfy this requirement. Indeed, the probability of finding the apparatus in the state, $m_{k}(2)$, and the object in the state $\ell_{v}(1)(v \neq k)$ should be zero (to preserve the one-to-one correspondence). This leads to the condition

$$
\alpha_{k k^{\prime}}=\alpha_{k} \delta_{k k^{\prime}}
$$

Thus, the form of the wave function after the measurement should be 


$$
|\Psi(1,2)\rangle=\sum_{k} \alpha_{k} \mid \ell_{k}(1) \mathrm{m}_{k}(2)>
$$

Now, according to the standard postulate of quantum mechanics, the probability of finding both the object and apparatus in the same state, $v$, is

$$
\left|<\ell_{v}(1) \mathrm{m}_{v}(2)\right||\Psi(1,2)>|^{2}=\left|\alpha_{v}\right|^{2}
$$

where we used the orthogonal relations (4.6).

The measurement process, embodied in the result (5.5) has established a statistical correlation between the states of the systems and the "pointers" in the apparatus; however, it has not emerged with the choice of a definite state. For this to happen, the wave function should experience discontinuous jumps: "the collapse of the wave function". This situation was recognized by von Neumann ${ }^{9}$ who made a distinction between the unitary evolution of the wave function, according to the Schrödinger equation, valid for isolated (closed) systems, and the "reduction" (collapse) of the wave function to a definite state.

\subsection{AXIOMATIC MEASUREMENT THEORY: THE PROJECTION POSTULATE}

von Neumann ${ }^{9}$ enunciated the so-called projection postulate that has become the axiomatic form of measurement theory. We follow the version of the postulate by Mahler and Weberruss. ${ }^{4}$ Consider the Hermitian operator, $\hat{A}$, with eigenvalue equation

$$
\hat{A}\left|a_{i}>=a_{i}\right| a_{i}>
$$

and projection operators, $\mathrm{P}_{m m}=\left|\mathrm{a}_{m}><\mathrm{a}_{m}\right|$

If a measurement represented by the $\hat{A}$-operator is carried out on an ensemble described by the density operator, $\hat{\rho}$, then after the measurement the system is in one of the pure states

$$
\hat{\rho}^{\prime}=\frac{1}{\mathrm{p}_{\mathrm{m}}} \hat{\mathrm{P}}_{\mathrm{mm}} \hat{\rho} \hat{\mathrm{P}}_{\mathrm{mm}}
$$

where $p_{m}$ is the probability of finding the result, $\mid a_{m}>$,

$$
\mathrm{p}_{m}=\mathrm{T}_{\mathrm{r}}\left\{\hat{\mathrm{P}}_{\mathrm{mm}} \hat{\rho}\right\}
$$

The result (5.8) is the mathematical formulation of von Neumann's projection postulate. An example is given in Appendix B. 
Note that the projection postulate is realized by the application of the "superoperators" $\hat{\mathrm{P}}_{m m n}(\bullet) \hat{\mathrm{P}}_{m m n}$. This operator can be expressed also in SU(n) algebra ${ }^{4}$. To this end, replace the general operator $\hat{\mathrm{A}}$ by $\hat{\mathrm{P}}_{m m}^{a}$ in equation (2.27) and use $\operatorname{Tr}_{r}\left\{\hat{\mathrm{P}}_{m, m}^{\alpha}\right\}=\hat{1}$ to get

$$
\hat{\mathrm{P}}_{\mathrm{rmm}}=\frac{1}{\mathrm{n}} \hat{\mathrm{i}}+\frac{1}{2} \sum_{\ell=1}^{n-1} \mathrm{X}_{\ell} \hat{\mathrm{W}}_{\ell}
$$

with

$$
\mathrm{X}_{\ell}=\mathrm{T}_{r}\left\{\hat{\mathrm{P}}_{m m} \hat{\mathrm{w}}_{\ell}\right\}=\mathrm{w}_{\ell}^{(m i)} .
$$

Then one obtains

$$
\hat{\mathrm{P}}_{m n}=\frac{1}{\mathrm{n}} \hat{1}+\frac{1}{2} \sum_{f=1}^{n-1} \mathrm{w}_{f}^{(m)} \hat{\mathrm{W}}_{f}
$$

and (see Appendix C)

$$
\begin{aligned}
& \hat{\mathrm{P}}_{m m} \hat{\mathrm{u}}_{i j} \hat{\mathrm{P}}_{m m}=\hat{\mathrm{P}}_{m m} \hat{\mathrm{v}}_{i j} \hat{\mathrm{P}}_{m m}=0 \\
& \hat{\mathrm{P}}_{m m} \hat{\mathrm{W}}_{\ell} \hat{\mathrm{P}}_{m m}=\hat{\mathrm{W}}_{\ell}{ }^{(m)} \hat{\mathrm{P}}_{m m} \\
& \hat{\mathrm{P}}_{m m} \hat{\mathrm{i}} \hat{\mathrm{P}}_{m m}=\hat{\mathrm{P}}_{m m} .
\end{aligned}
$$

By virtue of equation (5.12) up to (5.15), one obtains the relations below

$$
\hat{\mathrm{P}}_{m m} \hat{\rho} \hat{\mathrm{P}}_{m m}=\left\{\frac{1}{\mathrm{n}}+\frac{1}{2} \sum_{\ell=1}^{n-1} \mathrm{w}_{\ell}^{(m)} \mathrm{w}_{\ell}\right\} \mathrm{P}_{m m}
$$

where $\mathrm{w}_{\ell}$ is the expectation value

$$
\mathrm{w}_{\ell}=\mathrm{T}_{r}\left\{\hat{\rho} \hat{\mathrm{w}}_{\ell}\right\} \text {. }
$$

The probability of the outcome, $\mid \mathrm{m}>$, is now [use (5.12) and (5.17)]

$$
\mathrm{p}_{m}=\mathrm{T}_{r}\left\{\hat{\mathrm{P}}_{m m} \hat{\rho}\right\}=\frac{1}{\mathrm{n}}+\frac{1}{2} \sum_{\ell=1}^{n-1} \mathrm{~W}_{\ell}^{(m)} \mathrm{W}_{\ell}
$$


This page intentionally left blank. 


\section{NON SEPARABILITY: d'ESPAGNAT THEOREM}

Let two systems with wave functions

$$
\left.\left|\Phi(1)>=\sum_{m} \mathrm{a}\left(\mathrm{m}_{1}\right)\right| \mathrm{m}(1)>;\left|\Phi(2)>=\sum_{\mathrm{m}} \mathrm{a}\left(\mathrm{m}_{2}\right)\right| \mathrm{m}(2)\right\rangle
$$

be allowed to interact. Then, in general, the composite system wave function is given by the non-separable product:

$$
|\Psi(1,2)\rangle=a\left(m_{1} m_{2}\right) \mid m_{1} m_{2}>; a\left(m_{1} m_{2}\right) \neq a\left(m_{1}\right) a\left(m_{2}\right) .
$$

The corresponding density operator is

$$
\hat{\rho}=|\Psi\rangle\left\langle\Psi \left|=\sum_{m_{1} m_{2} \cdot m_{1}^{\prime} m_{2}^{\prime}} a\left(m_{1} m^{*}\left(m_{1}^{\prime} m_{2}^{\prime}\right)\left|m_{1} m_{2}\right\rangle<m^{\prime} m_{2}^{\prime} \mid .\right.\right.\right.
$$

Let us try to extract the subsystem $\mathrm{H}(1)$, for instance, by tracing out of existence the subsystem $H(2)$. To this end, we can use the concept of the reduced density operators developed in Section 4. Indeed, from (4.34) and (6.2) one gets:

$$
\left.\hat{\mathrm{R}}(1)=\mathrm{T}_{\mathrm{r} 2}\{\hat{\rho}\}=\sum_{\mathrm{m}_{2}}<\mathrm{m}_{2}|\hat{\rho}| \mathrm{m}_{2}\right\rangle=\sum_{\mathrm{m}_{1} \mathrm{~m}_{2} \mathrm{~m}_{1}^{\prime}} \mathrm{a}\left(\mathrm{m}_{2}\right) \mathrm{a}^{*}\left(\mathrm{~m}_{1}^{\prime} \mathrm{m}_{2}\right)\left|\mathrm{m}_{1}\right\rangle<\mathrm{m}_{1}^{\prime} \mid
$$

The result (6.4) does not allow us to write the reduced density as, $\hat{R}(1)=|\Phi(1)><\Phi(1)|$, unless that $|\Psi\rangle$ was indeed separable (i.e., $a\left(m_{1} m_{2}\right)=a\left(m_{1}\right) a\left(m_{2}\right)$. The results $(6.4)$ embodies the following theorem due to d'Espagnat ${ }^{10}$ : "If two subsystems have been interacting in the past, then it is generally not possible to define a state vector for any of the individual subsystems." It follows from this theorem that interaction leads to the entanglement of correlated subsystems and that it shows in the off-diagonal terms of the density matrix. 
This page intentionally left blank. 


\section{MEASUREMENTS ON COMPOSITE SYSTEMS}

\subsection{DEFINITION OF COMPOSITE SYSTEMS}

In this section we consider a composite system, $\mathrm{SU}(\mathrm{n}) \otimes \mathrm{SU}(\mathrm{n})$ in a state determined by two coherence vectors $\vec{\lambda}(1), \vec{\lambda}(2)$ with elements $\left(\lambda_{i}(1), \lambda_{k}(2)\right)$ and the correlation matrix $\mathrm{M}_{\mathrm{ij}}$. The density operator for this composite system, $\hat{\rho}(1,2)$ is given in equation $(4.23)$. Our goal is to evaluate the effect of a local measurement, for instance a measurement of $\hat{A}(2))$ in subsystem (2) on the composite system. In Section 4.5 we have learned that the proposed measurement on $\mathrm{H}(2)$ is represented by projection operators acting only on $\mathrm{H}(2)$. Following the developments in Section 4.5, we define the projection operator

$$
\hat{\mathrm{P}}_{m m}^{(2)}=\hat{\mathrm{1}} \otimes \hat{\mathrm{P}}(2)_{m m} ; \hat{\mathrm{P}}(2)_{m m}=|\mathrm{m}(2)><\mathrm{m}(2)| \text {. }
$$

When the system is described by the density operator, $\hat{\rho}(1,2)$, in accordance with axiomatic measurement theory, the probability of finding the result, $a_{m}^{(2)}$ is given by

$$
\mathrm{p}_{\mathrm{m}}(2)=\mathrm{T}_{r}\left\{\hat{\mathrm{P}}_{m m}^{(2)} \hat{\rho}\right\}=\mathrm{T}_{r 2}\left\{\hat{\mathrm{P}}_{\mathrm{mm}}(2) \hat{\mathrm{R}}(2)\right\}
$$

where, $\hat{R}(2)$, is the reduced operator defined in (4.35). After the measurement, the system reduces to

$$
\hat{\mathrm{p}}_{\mathrm{m}}(2) \hat{\rho}^{\prime}=\hat{\mathrm{P}}_{\mathrm{mm}}(2) \quad \hat{\rho} \hat{\mathrm{P}}_{\mathrm{mm}}(2)
$$

Inserting in (7.3) the development of the density operator, $\hat{\rho}(1,2)$ in terms of SU(n) generators, equation (4.23), one obtains $\left(n_{1}=n_{2}=n\right)$

$$
\begin{aligned}
& \hat{\mathrm{P}}_{\mathrm{m}}(2) \hat{\rho}^{\prime}=\hat{\mathrm{P}}_{\mathrm{mm}}(2)\left\{\frac{1}{\mathrm{n}^{2}} \hat{\mathrm{l}}(1) \otimes \hat{\mathrm{l}}(2)+\frac{1}{2 \mathrm{n}} \sum_{j=1}^{s} \lambda_{,}(1)\left[\hat{\lambda}_{j}(1) \otimes \hat{\mathrm{l}}(2)\right]\right. \\
& \left.+\frac{1}{2 \mathrm{n}} \sum_{k=1}^{s} \lambda_{k}(2)\left[\hat{\mathrm{l}}(1) \otimes \hat{\lambda}_{k}(2)\right]+\frac{1}{4} \sum_{j k=1}^{s} \mathrm{~K}_{j k}(1,2)\left[\hat{\lambda}_{j}(1) \otimes \hat{\lambda}_{k}(2)\right]\right\} \hat{\mathrm{P}}_{\mathrm{mm}}(2)
\end{aligned}
$$

After some manipulations (see Appendix D) one obtains

$$
\begin{aligned}
& \hat{\mathrm{p}}_{\mathrm{m}}(2) \hat{\rho}^{\prime}=\frac{1}{\mathrm{n}^{2}} \hat{\mathrm{1}}(1) \otimes \hat{\mathrm{P}}_{\mathrm{mm}}(2)+\frac{1}{2 \mathrm{n}} \sum_{j=1}^{s} \lambda_{j}(1)\left[\hat{\lambda}_{j}(1) \otimes \hat{\mathrm{P}}_{\mathrm{mm}}(2)\right. \\
& +\frac{1}{2 \mathrm{n}} \sum_{\rho=1}^{n-1} \mathrm{w}_{\ell}(2) \mathrm{w}_{\rho}^{(m)}\left[\hat{1}(1) \otimes \hat{\mathrm{P}}_{\mathrm{mm}}(2)\right] \\
& +\frac{1}{4} \sum_{j}^{s} \sum_{\rho=1}^{n-1} \mathrm{~K}_{j}, s^{\prime}+\ell \mathrm{w}_{r}^{(m)}\left[\hat{\lambda}_{j}(1) \otimes \hat{\mathrm{P}}_{\mathrm{mm}}(2)\right.
\end{aligned}
$$


where it is to be reminded that the expectation values, $w_{\ell}(2)$, are given, see equation $(5.17)$ by

$$
\mathrm{w}_{f}(2)=\mathrm{T}_{r}\left\{\hat{\rho} \hat{\mathrm{w}}_{\varphi}(2)\right\}
$$

and, $s^{\prime}=n^{2}-n$.

In the next step, we use the representation (5.12) of the projection operators in terms of the $\mathrm{SU}(\mathrm{n})$ generators, $\hat{\mathrm{w}}_{\ell}$ :

$$
\hat{\mathrm{P}}_{\mathrm{mm}}(2)=\frac{1}{\mathrm{n}} \hat{\mathrm{l}}(2)+\frac{1}{2} \sum_{i=1}^{n-1} \mathrm{w}_{\ell}^{(m)} \hat{\mathrm{w}}_{\ell}(2)
$$

and equation (5.18) for the probability $\mathrm{p}_{\mathrm{m}}(2)$ :

$$
\mathrm{p}_{\mathrm{tn}}(2)=\frac{1}{\mathrm{n}}+\frac{1}{2} \sum_{i=1}^{n-1} \mathrm{w}_{f}^{(m)} \mathrm{w}_{,}(2)
$$

to obtain from equations $(7.5,7.7$, and 7.8) (see Appendix D).

$$
\begin{aligned}
& \hat{\rho}^{\prime}=\frac{1}{\mathrm{n}^{2}} \hat{\mathrm{l}}(1) \otimes \hat{\mathrm{l}}(2)+\frac{1}{2 \mathrm{n}} \sum_{\ell=1}^{n-1} \mathrm{w}_{\ell}^{(m)}\left[\hat{\mathrm{l}}(1) \otimes \hat{\mathrm{w}}_{\ell}(2)\right] \\
& +\frac{1}{2 p_{m}(2)}\left\{\frac{1}{\mathrm{n}} \sum_{j}^{s} \mathrm{~K}_{j}^{(1)}(1,2)\left[\hat{\lambda}_{j}(1) \otimes \hat{\mathrm{l}}(2)\right]+\frac{1}{2} \sum_{p=1}^{n-1} \mathrm{~K}_{j l}^{(2)}(1,2)\left[\hat{\lambda}_{j} \otimes \hat{\mathrm{w}}_{f}(2)\right]\right\}
\end{aligned}
$$

where

$$
\begin{aligned}
& \mathrm{K}_{j}{ }^{(1)}=\frac{1}{\mathrm{n}} \lambda_{j}(1)+\frac{1}{2} \sum_{\ell=1}^{n-1} \mathrm{~K}_{j, s^{\prime}+\ell^{\prime}} \mathrm{W}_{\ell}{ }^{(m)} \\
& \mathrm{K}_{j l}^{(2)}=\frac{1}{\mathrm{n}} \lambda_{j}(1) \mathrm{w}_{\ell}{ }^{(m)}+\frac{1}{2} \sum_{\ell^{\prime}=1}^{n-1} \mathrm{~K}_{j, x^{\prime}+\ell^{\prime}} \quad \mathrm{W}_{\ell^{\prime}}{ }^{(m)} \mathrm{w}_{\ell}{ }^{(m)} .
\end{aligned}
$$

The question now is to ask for the changes in the coherence vectors of the two subspaces. $\vec{\lambda}(1), \vec{\lambda}(2)$, after a measurement performed on $H(2)$. To answer this question, we start from the relation (4.24) written in terms of the density operator, $\hat{\rho}^{\prime}$, for the collapsed state:

$$
\lambda^{\prime}{ }_{j}(1)=\operatorname{Tr}\left\{\hat{\rho}^{\prime} \hat{\lambda}_{j}(1) \otimes \hat{1}(2)\right\}
$$

Insertion of equation (7.9) for $\hat{\rho}^{\prime}$ into (7.12) yields 


$$
\begin{aligned}
& \lambda_{j}^{\prime}(1)=T_{r}\left\{\hat { \lambda } _ { j } ( 1 ) \left[\frac{1}{n^{2}} \hat{1}(1) \otimes \hat{1}(2)+\frac{1}{2 n} \sum_{i=1}^{n-1} w_{r}^{(m)}\left[\hat{l}(1) \otimes \hat{\lambda}_{s+r}^{(2)}\right]\right.\right.
\end{aligned}
$$

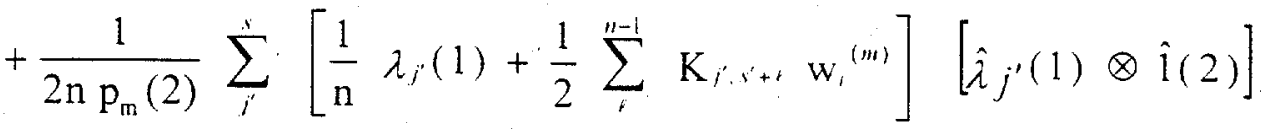

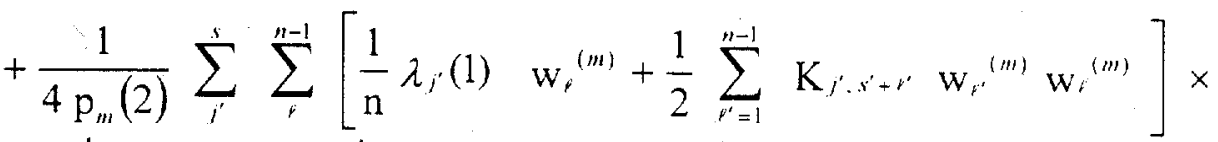

$$
\begin{aligned}
& \times\left\lfloor\hat{\lambda}_{j^{\prime}}(1) \otimes \hat{\lambda}_{s^{\prime}+f}\right\rfloor
\end{aligned}
$$

where we used [see Eq. (2.23)], $\hat{\mathrm{w}}_{\ell}(2)=\hat{\lambda}_{s+e}(2)$. To work out the trace relations in Eq. (7.13), we need to recall the following properties of the coherence vectors [Eqs. (2.22), $(4.21),(4.22)$, and (4.14)]

$$
\begin{aligned}
& \operatorname{Tr}\left\{\lambda_{j}(1) \otimes \hat{1}(2)\right\}=0 ; \operatorname{Tr}_{r}\left\{\hat{\lambda}_{j}(1) \hat{\lambda}_{k}(1)\right\}=2 \delta_{j k} \\
& \operatorname{Tr}\left\{\hat{\lambda}_{j}(1) \otimes \hat{\lambda}_{k}(2)\right\}=0 \\
& \operatorname{Tr}\left\{\left[\lambda_{j}(1) \otimes \hat{\lambda}_{k}(2)\right]\left[\hat{\lambda}_{l}(1) \otimes \hat{\lambda}_{m}(2)\right]\right\}=4 \delta_{j l} \delta_{k m}
\end{aligned}
$$

After use of the trace relations (7.14) up to (7.16), one obtains

$$
\lambda_{j}^{\prime}(1)=\frac{1}{\mathrm{p}_{m}(2)}\left(\frac{1}{\mathrm{n}} \lambda_{j}(1)+\frac{1}{2} \sum_{\ell=1}^{n-1} \mathrm{~K}_{j s^{\prime}+} \mathrm{w}_{p}{ }^{\left(m_{2}\right)}\right)
$$

In the absence of interaction, the correlation tensor [see (4.26), (2.23)] becomes the product (remember $\underset{s+\ell}{\lambda(2)}=w_{\ell}$ )

$$
\mathrm{K}_{j s^{\prime}+\ell}=\lambda_{j}(1) \mathrm{w}_{\ell}(2)
$$

Thus, (7.17) now reads:

$$
\lambda_{j}^{\prime}(1)=\frac{\lambda_{j}(1)}{\mathrm{p}_{m}(2)}\left\{\frac{1}{\mathrm{n}}+\frac{1}{2} \sum_{\rho=1}^{n-1} \mathrm{w}_{\ell}{ }^{\left(m_{2}\right)} \mathrm{w}_{\ell}(2)\right\}
$$

and by virtue of $(7.8)$ for $\mathrm{p}_{\mathrm{m}}(2)$ one obtains the expected result that the subsystem, $\mathrm{H}(1)$, remains untouched by the measurement on $\mathrm{H}(2)\left(\lambda_{j}^{\prime}(1)=\lambda_{j}(1)\right.$. We now turn to find out the new value of the coherence vector $\bar{\lambda}(2)$. Start from 


$$
\lambda^{\prime},(2)=\mathrm{T}_{r}\left\{\hat{\rho}^{\prime}(1,2) \hat{\mathrm{i}}(1) \otimes \hat{\lambda}_{j}(2)\right\}
$$

insert Eq. (7.9) and follow the same steps as before.

$$
\lambda_{j}^{\prime}(2)=\sum_{i=1}^{n-1} \mathrm{w}_{\ell}^{(m)} \delta_{j, s^{\prime+e}}
$$

For subsystems that had interacted, the coherence vector of the non-acted upon subsystem evolves according to the result (7.17): "Local measurements induce non-local effects",

\subsection{CONDITIONAL PROBABILITIES}

The probability, $p_{1 m}(1)$, of finding the subsystem (1) in the $m_{1}$-th state after having performed a measurement on subsystem (2) (i.e., the conditional probability, $\mathrm{p}_{1}\left(\mathrm{~m}_{1} \mid \mathrm{m}_{2}\right)$ is given by [see Eq. (7.2)]

$$
\mathrm{p}_{1}\left(\mathrm{~m}_{1} \mid \mathrm{m}_{2}\right)=\mathrm{T},\left\{\hat{\mathrm{P}}_{\mathrm{mm}}(1) \hat{\rho}^{\prime}\right\}
$$

in terms of the "collapsed" density operator, Eq. (7.9). Note that the projection operator, $\hat{\mathrm{P}}_{m m}(1)$, is given in terms of the coherence vectors $\vec{\lambda}^{\prime}(1), \vec{\lambda}^{\prime}(2)$ after the measurement, then Eq. (7.7) is rewritten as:

$$
\hat{\mathrm{P}}_{\mathrm{mm}}(1)=\frac{1}{\mathrm{n}} \hat{1}(1)+\frac{1}{2} \sum_{\ell=1}^{n-1} \mathrm{w}_{\ell}^{(m)} \hat{\lambda}_{\gamma^{\prime}+\ell}^{\prime}(1) \quad ;\left(\hat{\lambda}_{s^{\prime}+\ell}(1)=\hat{\mathrm{w}}_{\ell}(1)\right) .
$$

Insert (7.23) into (7.22) and use

$$
\mathrm{T}_{r}\left\{\hat{\rho}^{\prime}\right\}=1 ; \quad \lambda_{s^{\prime}+\ell}^{\prime}(1)=\mathrm{T}_{r}\left\{\hat{\rho}^{\prime} \hat{\lambda}_{s^{\prime}+\varphi}^{\prime}(1)\right\}
$$

to get

$$
p_{1}\left(m_{1} \mid m_{2}\right)=\frac{1}{n}+\frac{1}{2} \sum_{\ell=1}^{n-1} w_{\ell}{ }_{\ell}^{\left(m_{l}^{\prime}\right)} \lambda_{N^{\prime}+\ell}(1)
$$

Next use (7.17) to get

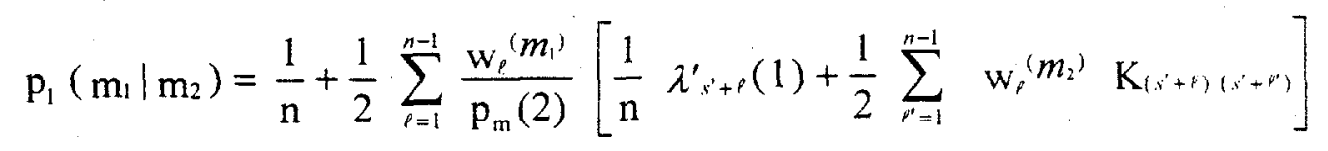

where, $\mathrm{p}_{\mathrm{m}}(2)$, is given by Eq. (7.8). 
Finally, the joint probability is given by

$$
\mathrm{p}\left(\mathrm{m}_{1}, \mathrm{~m}_{2}\right)=\mathrm{p}\left(\mathrm{m}_{2}\right) \mathrm{p}_{1}\left(\mathrm{~m}_{1} \mid \mathrm{m}_{2}\right)=\mathrm{p}\left(\mathrm{m}_{1}\right) \mathrm{p}\left(\mathrm{m}_{2} \mid \mathrm{m}_{1}\right)
$$

\subsection{CORRELATION FUNCTION}

Consider two $\mathrm{SU}(\mathrm{n})$ subsystems ${ }^{4}$ defineed in their respective $\mathrm{H}(v)$ spaces and specify two measurement operators, $\hat{A}(v)(v=1,2)$. These operators can be expressed in terms of the $\mathrm{SU}(\mathrm{n})$ generators as shown in Eq. (2.26). Their respective expectation values $\langle\mathrm{A}\rangle$ are given by Eq. (2.38); i.e.,

$$
<\hat{\mathrm{A}}>=\operatorname{T}_{r}\{\hat{\rho} \hat{\mathrm{A}}(v)\}=\mathrm{T}_{r}\left\{\hat{\rho} \mathrm{T}_{r}\{\hat{\mathrm{A}}(v)\}+\frac{1}{2} \sum_{j=1}^{s} \mathrm{~A}_{i}(v) \mathrm{T}_{r}\left\{\hat{\rho} \hat{\lambda}_{i}(v)\right\}\right\} .
$$

Now since $\operatorname{Tr}\{\hat{\rho}\}=1(2.41)$ and the definition (2.42) of the expectation value of the $\mathrm{j}$-th generator, one obtains:

$$
\langle\hat{\mathrm{A}}(v)\rangle=\frac{1}{\mathrm{n}} \operatorname{Tr}\{\hat{\mathrm{A}}(v)\}+\frac{1}{2} \sum_{j=1}^{s} \mathrm{~A}_{j}(v) \lambda_{j}(v)
$$

where recall $(2.26)$

$$
\mathrm{A}_{j}(v)=\mathrm{T}_{r}\left\{\hat{\mathrm{A}}(v) \hat{\lambda}_{\mathrm{j}}(v)\right\}
$$

and $s=n^{2}-1$.

Next consider a $S U(n) \otimes S U(n)$ composite system and form the representation of the direct product $A(1) \otimes A(2)$, in the manner shown in Section 4.3. In terms of the $S U(n)$ generators, one obtains

$$
\begin{aligned}
\hat{\mathrm{A}}(1) & \otimes \hat{\mathrm{A}}(2)=\frac{1}{\mathrm{n}^{2}} \mathrm{~T}_{r 1}\{\hat{\mathrm{A}}(1)\} \mathrm{T}_{r 2}\{\hat{\mathrm{A}}(2)\} \\
& +\frac{1}{2 \mathrm{n}} \mathrm{T}_{r 1}\{\hat{\mathrm{A}}(1)\} \sum_{j}^{s} \mathrm{~A}_{j}(2)\left[\hat{\mathrm{l}}(1) \otimes \hat{\lambda}_{j}(2)\right] \\
& +\frac{1}{2 \mathrm{n}} \mathrm{T}_{r 2}\{\hat{\mathrm{A}}(2)\} \sum_{j}^{s} \mathrm{~A}_{j}(1)\left[\hat{1}(1) \otimes \hat{\lambda}_{j}(2)\right] \\
& +\frac{1}{4} \sum_{j k}^{s} \mathrm{~A}_{j}(1) \mathrm{A}_{k}(2)\left[\hat{\lambda}_{j}(1) \otimes \hat{\lambda}_{k}(2)\right] .
\end{aligned}
$$

The quantum mechanical correlation function is defined as the expectation value of the tensor product of operators: 


$$
\begin{aligned}
\langle\hat{\mathrm{A}}(1) \otimes \hat{\mathrm{A}}(2)\rangle & =\mathrm{T}_{r}\{\hat{\rho}(1,2) \hat{\mathrm{A}}(1) \otimes \hat{\mathrm{A}}(2)\} \\
& =\frac{1}{\mathrm{n}^{2}} \mathrm{~T}_{r 1}\{\hat{\mathrm{A}}(1)\} \mathrm{T}_{r 2}\{\hat{\mathrm{A}}(2)\} \mathrm{T}_{r}[\hat{\mathrm{i}}(1 ; \otimes \hat{\mathrm{i}}(2)] \\
& +\frac{1}{2 \mathrm{n}} \mathrm{T}_{r 1}\{\hat{\mathrm{A}}(1)\} \sum_{j=1}^{s} \mathrm{~A}_{j}(2) \mathrm{T}_{r}\left\{\hat{\mathrm{I}}(1) \otimes \hat{\lambda}_{j}(2)\right\} \\
& +\frac{1}{2 \mathrm{n}} \mathrm{T}_{r 2}\{\hat{\mathrm{A}}(2)\} \sum_{j=1}^{s} \mathrm{~A}_{j}(1) \mathrm{T}_{r}\left\{\hat{\lambda}_{j}(1) \otimes \hat{\mathrm{i}}(2)\right\} \\
& +\frac{1}{4} \sum_{j k}^{s} \mathrm{~A}_{j}(1) \mathrm{A}_{k}(2) \mathrm{T}_{r}\left[\hat{\lambda}_{j}(1) \otimes \hat{\lambda}_{k}(2)\right]
\end{aligned}
$$

Now use Eqs. (4.20), (2.42), and (4.25) to get

$$
\begin{aligned}
\langle\hat{\mathrm{A}}(1) \otimes \hat{\mathrm{A}}(2)\rangle & =\frac{1}{\mathrm{n}^{2}} \mathrm{~T}_{r 1}\{\hat{\mathrm{A}}(1)\} \mathrm{T}_{r}\{\hat{\mathrm{A}}(2)\} \\
& +\frac{1}{2 \mathrm{n}} \mathrm{T}_{r 1}\{\hat{\mathrm{A}}(1)\} \sum_{j}^{s} \mathrm{~A}_{j}(2) \lambda_{j}(2) \\
& +\frac{1}{2 \mathrm{n}} \mathrm{T}_{r 2}\{\hat{\mathrm{A}}(2)\} \sum_{j}^{s} \mathrm{~A}_{j}(1) \lambda_{j}(1) \\
& +\frac{1}{4} \sum_{j k}^{s} \mathrm{~K}_{j k}(1,2) \mathrm{A}_{j}(1) \mathrm{A}_{k}(2) .
\end{aligned}
$$

Comparison of the above result with [(7.29) and use of Eq. (4.27)] for the correlation matrix, $\mathrm{M}_{\mathrm{ik}}$ yields

$$
\langle\hat{\mathrm{A}}(1) \otimes \hat{\mathrm{A}}(2)\rangle=\langle\mathrm{A}(1)\rangle\langle\mathrm{A}(2)\rangle+\mathrm{C}_{A A}
$$

where

$$
\mathrm{C}_{A A}=\frac{1}{4} \sum_{j, k=1}^{s} \mathrm{~A}_{j}(1) \mathrm{M}_{j k}(1,2) \mathrm{A}_{k}(2) .
$$

The result (7.34) shows that a correlation function of second order can be decomposed into the product of the expectation values of the individual systems (correlation of first order) and an additional second order correlation, (AA).

\subsection{THE SU(2) $\otimes$ SU(2) SYSTEM}

For the upcoming discussions on the EPR-paradox and Bell's; inequalities it becomes convenient to replay the preceding results for a $S U(2) \otimes S U(2)$ composite system. To this end, consider the singlet state of two particles of one-half spin: 


$$
\mid \Psi>=\frac{1}{\sqrt{2}}\left[\left|\mathrm{~m}_{1}(1)>\right| \mathrm{m}_{2}(2)>-\left|\mathrm{m}_{1}(2)>\right| \mathrm{m}_{2}(1)>\right]
$$

where $\left|m_{1}(v)\right\rangle, \mid m_{2}(v)>,(v=1,2)$ denote spin eigenfunctions with spin "up" $(v=2)$ and spin "down" $(\nu=1)$. The singlet state (7.36) represents an entangled state since it cannot be reduced to a product of states. Either subsystem can be in either of the "up" and "down" states. The $\mathrm{SU}(2)$ operator $\hat{\mathrm{w}}_{\ell}$ (Eq. 2.25) acts on the m-states as:

$$
\hat{\mathrm{w}}_{f}\left|\mathrm{~m}_{1}(v)\right\rangle=\mathrm{w}_{f}{ }^{(v)} \mathrm{m}_{1}(v) ; \quad \hat{\mathrm{w}}_{f}\left|\mathrm{~m}_{2}(v)>=\mathrm{w}_{f}{ }^{(v)}\right| \mathrm{m}_{2}(v)>
$$

where the eigenvalues, $w_{\ell}^{(\nu)}$, are given by Eqs. (2.29) up to (2.31). The components of the coherent vector are the expectation values of the generator $\hat{\lambda}_{j}$, i.e. (Eq. 2.42)

$$
\lambda_{j}=\mathrm{T}_{r}\left\{\begin{array}{ll}
\hat{\rho} & \hat{\lambda}_{j}
\end{array}\right\}
$$

The SU(2) group generators are [Eqs. (2.23) (2.25)]

$$
\hat{\lambda}_{1}=\hat{\mathrm{u}}_{12}=\hat{\mathrm{P}}_{12}+\hat{\mathrm{P}}_{21} ; \hat{\lambda}_{2}=\hat{v}_{12}=i\left(\hat{\mathrm{P}}_{12}-\hat{\mathrm{P}}_{21}\right) ; \hat{\lambda}_{3}=\hat{\mathrm{w}}_{3}=-\left(\hat{\mathrm{P}}_{11}-\hat{\mathrm{P}}_{22}\right)
$$

where the projection operators are given by

$$
\hat{\mathrm{P}}_{i j}(1)=\left|\mathrm{m}_{1}(j)><\mathrm{m}_{1}(i)\right| ; \quad \hat{\mathrm{P}}_{i j}(2)=\left|\mathrm{m}_{2}(j)><\mathrm{m}_{2}(i)\right|
$$

and the density operator is in terms of the above set of projection operators

$$
\begin{aligned}
\hat{\rho}(1,2)=|\Psi\rangle\langle\Psi| & =\frac{1}{2}\left\{\hat{\mathrm{P}}_{11}(1) \hat{\mathrm{P}}_{22}(2)-\hat{\mathrm{P}}_{12}(1) \hat{\mathrm{P}}_{21}(2)\right. \\
& -\hat{\mathrm{P}}_{21}(1) \hat{\mathrm{P}}_{12}(2)+\hat{\mathrm{P}}_{22}(1) \hat{\mathrm{P}}_{12}(2) .
\end{aligned}
$$

We now work out the $\mathrm{j}=1$; component of the coherence vector for the subsystem $\mathrm{H}(1)$, i.e., insert (7.39) and (7.41) into (7.38) 


$$
\begin{aligned}
\lambda_{1}(1) & =\frac{1}{2} \mathrm{~T}_{r}\left\{\hat{\mathrm{P}}_{11}(1) \hat{\mathrm{P}}_{12}(1) \hat{\mathrm{P}}_{22}(2)-\hat{\mathrm{P}}_{12}(1) \hat{\mathrm{P}}_{12}(1) \hat{\mathrm{P}}_{21}(2)-\hat{\mathrm{P}}_{21}(1) \hat{\mathrm{P}}_{12}(1) \hat{\mathrm{P}}_{12}(2)\right. \\
& +\hat{\mathrm{P}}_{22}(1) \hat{\mathrm{P}}_{12}(1) \hat{\mathrm{P}}_{12}(2) \\
& +\hat{\mathrm{P}}_{11}(1) \hat{\mathrm{P}}_{12}(1) \hat{\mathrm{P}}_{22}(2)-\hat{\mathrm{P}}_{12}(1) \hat{\mathrm{P}}_{21}(1) \hat{\mathrm{P}}_{21}(2)-\hat{\mathrm{P}}_{21}(1) \hat{\mathrm{P}}_{21}(1) \hat{\mathrm{P}}_{12}(2) \\
& \left.+\hat{\mathrm{P}}_{22}(1) \hat{\mathrm{P}}_{21}(1) \hat{\mathrm{P}}_{11}(2)\right\}
\end{aligned}
$$

Now use (2.17) to get

$$
\begin{aligned}
\lambda_{1}(1) & =\frac{1}{2} \operatorname{T}\left\{\hat{\mathrm{P}}_{12}(1) \hat{\mathrm{P}}_{22}(2)-0-\hat{\mathrm{P}}_{12}(2) \hat{\mathrm{P}}_{22}(1)+0\right. \\
& +\hat{\mathrm{P}}_{12}(1) \hat{\mathrm{P}}_{22}(2)-\hat{\mathrm{P}}_{11}(1) \hat{\mathrm{P}}_{21}(2)-0+\hat{\mathrm{P}}_{21}(1) \hat{\mathrm{P}}_{11}(2)
\end{aligned}
$$

Use Eq. (2.19) (i.e., $T_{r}\left\{P_{k \ell}\right\}=\delta_{k \ell}$ ) to obtain $\lambda_{1}(1)=0$. Following a similar procedure for the other components and subsystems (2), one obtains

$$
\lambda_{j}(1)=\lambda_{j}(2)=0
$$

Next, we calculate the correlation tensor $K_{j k}(1,2)$ [see Eq. (4.26)]. We illustrate this calculation for the diagonal term, $\mathrm{K}_{11}$, which from (4.26) is written as:

$$
\mathrm{K}_{11}(1,2)=\left\langle\hat{\lambda}_{1}(1) \hat{\lambda}_{1}(2)\right\rangle=\operatorname{Tr}\left\{\hat{\rho}\left\lfloor\hat{\lambda}_{1}(1) \otimes \hat{\lambda}_{1}(2)\right\rfloor\right\}
$$

where from (7.39)

$$
\begin{aligned}
\hat{\lambda}_{1}(1) \otimes \hat{\lambda}_{1}(2) & =\left\{\hat{\mathrm{P}}_{12}(1) \otimes \hat{\mathrm{P}}_{12}(2)+\hat{\mathrm{P}}_{12}(1) \otimes \hat{\mathrm{P}}_{21}(2)\right. \\
& \left.+\hat{\mathrm{P}}_{21}(1) \otimes \hat{\mathrm{P}}_{12}(2)+\hat{\mathrm{P}}_{21}(1) \otimes \hat{\mathrm{P}}_{21}(2)\right\}
\end{aligned}
$$

Insert the density operator (7.41) and Eq. (7.46) into (7.45), then after repeated use of Eq. (2.17) yields, $K_{11}(1,2)=-1$ and in general

$$
\mathrm{K}_{i j}=-\delta_{i j}
$$

The coherence vectors after the measurement on $\mathrm{H}(2)$ are given by Eqs. (7.17), (7.21), and. $\mathrm{K}_{\mathrm{ij}}$ by (7.47) (recall that the coherence vectors were zero before the measurement). We got from $(7.17)(\mathrm{n}=2)$ :

$$
\begin{aligned}
& \lambda_{j+}^{\prime}(1)=\frac{1}{2 \mathrm{p}_{m_{2}}(1)} \sum_{\ell=1}^{1} \mathrm{~K}_{j, 2+\ell} \mathrm{w}_{\ell}{ }^{(1)}=-\frac{\mathrm{w}_{1}{ }^{(1)}}{2 \mathrm{P}_{m_{2}}{ }^{(1)}} \delta_{j 3} \\
& \lambda^{\prime},(1)=\frac{1}{2 \mathrm{p}_{m_{2}}(2)} \sum_{\ell=1}^{1} \mathrm{~K}_{j, 2+\ell} \mathrm{w}_{\ell}{ }^{(2)}=-\frac{\mathrm{w}_{1}{ }^{(2)}}{2 \mathrm{P}_{m_{2}}{ }^{(2)}} \delta_{j 3}
\end{aligned}
$$


where, $\mathrm{p}_{\mathrm{m} 2}(1)$ is the probability that after a measurement in $\mathrm{H}(2)$ one finds the state $\mathrm{m}_{2}(1)$ (spin "down") and $\mathrm{p}_{\mathrm{m} 2}(2)$ the probability of finding $\mathrm{H}(2)$ in the state $\mathrm{m}_{2}(2)$ (spin "up").

Correspondingly, $\lambda^{\prime} j_{+}(1)$, is the coherence vector, $\vec{\lambda}_{+}(1)$, associated with the state $\mathrm{m}_{1}(2)$ and $\vec{\lambda}_{-}(1)$, with the state $\mathrm{m}_{1}(1)$. From Eq. (7.8) one obtains (see Appendix D)

$$
\begin{aligned}
& \mathrm{p}_{m_{2}}(1)=\frac{1}{2}\left(1+\mathrm{w}_{1}{ }^{(1)} \mathrm{w}_{1}{ }^{(2)}\right)=\frac{1}{2}\left(1+\mathrm{w}_{1}{ }^{(1)} \lambda_{3}(2)\right)=\frac{1}{2} \\
& \mathrm{p}_{m_{2}}(2)=\frac{1}{2}\left(1+\mathrm{w}_{1}{ }^{(2)} \lambda_{3}{ }^{(2)}\right)=\frac{1}{2}
\end{aligned}
$$

where we used the fact that $\bar{\lambda}(2)=0$.

We can now calculate the conditional probabilities, $\mathrm{p}\left(\mathrm{m}_{1}(1) \mid \mathrm{m}_{2}(2)\right), \mathrm{p}\left(\mathrm{m}_{1}(2) \mid \mathrm{m}_{2}(1)\right)$, $\mathrm{p}\left(\mathrm{m}_{1}(1) \mid \mathrm{m}_{2}(1)\right)$, and $\mathrm{p}\left(\mathrm{m}_{1}(2) \mid \mathrm{m}_{2}(2)\right)$

$$
\begin{aligned}
& \mathrm{p}\left(\mathrm{m}_{\mathrm{i}}(1) \mid \mathrm{m}_{2}(2)\right)=\frac{1}{2}\left[\begin{array}{ll}
1+\mathrm{w}_{1}{ }^{(1)} & \lambda^{\prime}{ }_{3-}
\end{array}\right]=\frac{1}{2}\left[\begin{array}{ll}
1-\mathrm{w}_{1}{ }^{(1)} & \mathrm{w}_{1}{ }^{(2)}
\end{array}\right] \\
& \mathrm{p}\left(\mathrm{m}_{1}(2) \mid \mathrm{m}_{2}(1)\right)=\frac{1}{2}\left[1+\mathrm{w}_{1}^{(2)} \lambda_{3+}^{\prime}\right]=\mathrm{p}\left(\mathrm{m}_{\mathrm{l}}(1) \mid\left(\mathrm{m}_{2}(2)\right.\right.
\end{aligned}
$$

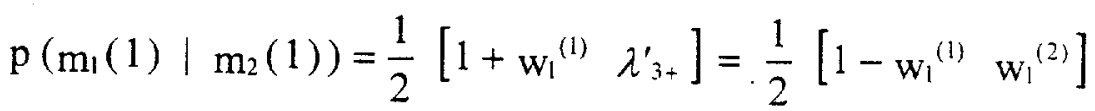

$$
\begin{aligned}
& \mathrm{p}\left(\mathrm{m}_{1}(2) \mid \mathrm{m}_{2}(2)\right)=\frac{1}{2}\left[\begin{array}{ll}
1+\mathrm{w}_{1}^{(2)} & \lambda^{\prime}{ }_{3-}
\end{array}\right]=\frac{1}{2}\left[\begin{array}{ll}
1-\mathrm{w}_{1}^{(2)} & \mathrm{w}_{1}^{(2)}
\end{array}\right]
\end{aligned}
$$

where the eigenvalues $\mathrm{w}_{1}{ }^{(v)}(v=1,2)$ are from (2.29) up to $(2.31)$

$$
\mathrm{w}_{1}{ }^{(1)}=-1 ; \quad \mathrm{w}_{1}{ }^{(2)}=1
$$

Inserting (7.56) into (7.52) up to (7.55) one obtains

$$
\begin{aligned}
& \mathrm{p}\left(\mathrm{m}_{1}(1) \mid \mathrm{m}_{2}(2)\right)=\mathrm{p}\left(\mathrm{m}_{2}(2) \mid \mathrm{m}_{1}(1)\right)=1 \\
& \mathrm{p}\left(\mathrm{m}_{1}(1) \mid \mathrm{m}_{2}(1)\right)=\mathrm{p}\left(\mathrm{m}_{1}(2) \mid \mathrm{m}_{2}(2)\right)=0
\end{aligned}
$$

in agreement with common sense. 
The joint probability will then be

$$
p\left(m_{1}(1), m_{2}(2)\right)=p\left(m_{1}(2), m_{2}(1)\right)=\frac{1}{2}
$$

For a factorable state, the correlation tensor is zero, hence all the conditional probabilities become one-half and the joint probabilities become one-fourth in agreement with the fact that there are four possible outcomes in this case. 


\section{DECOHERENCE}

Decoherence is the "bête noire" 11 of entanglement. Indeed, so far we have been dealing with "closed" systems isolated from the environment with the exception perhaps of externally applied fields. Information on a quantum object is, in contrast, obtained only by

measurement that necessarily involves interaction with the environment and hence the study of "open" systems. Clearly, one can consider the interaction of an object with the rest of the Universe as being again a closed system described by an entangled density operator with non-diagonal terms arising from their interaction. The effect of the environment was already discussed in classical mechanics by Borel who, in 1914, pointed out to the large "effect" of a small mass of a few grams, as far away as the star Sirius on the trajectories of air molecules have on earth." "To evaluate the problems associated with the object environment closed system, consider the processes involved in the detection of a nuclear particle by a Geiger counter and its associated electronics. First, the nuclear particle induces an ionization process, clearly of quantum mechanical nature, afterwards the secondary particles diffuse within the gas under an electric potential gradient and collect on a wire. Through appropriate electronics, the charge produced by the ionization process gets transformed into a pulse that produces a "click" in a macro-object. Anybody brave enough to model this process will be faced with the question of setting the so-called "Heisenberg cut" defining the line between the quantum and classical descriptions. Worse yet, even at the quantum-mechanical level it will be difficult to split the total Hamiltonian into unperturbed and interacting parts. One can get some good answers by an approximate description of the "open" system as a "closed" system consisting of the quantum object interacting with a "bath" modeled as a many degree of freedom system with a quasi-continuous spectrum (see Ref. 4, page 242) for a

mathematical description of such a model. The wave functions of the quantum object and the "bath" become entangled. Then due to the large reservoir of microscopic variables, when the density matrix is traced over the environmental components, one induces a fast dissipation of coherence by an eventual cancellation of its non-diagonal elements. "I 
This page intentionally left blank. 


\section{THE AHARONOV-BOHM EFFECT}

In 1959, Aharonov and Bohm ${ }^{12}$ demonstrated that a charged particle can be influenced by electromagnetic potentials even when the electromagnetic fields themselves are null (The $A B$ Effect). Indeed, if $|\Psi\rangle$ is a solution of the wave equation for a charged particle in the absence of an electromagnetic field, then in the presence of a static magnetic field, the wave function evolves into:

$$
|\Psi>=| \Psi_{\prime \prime}>\exp \left\{i\left(\frac{2 \pi e}{h c}\right) \int_{\vec{r}_{0}}^{\bar{r}} \overrightarrow{\mathrm{A}} \cdot \mathrm{d} \overrightarrow{\mathrm{s}}\right\}
$$

where, $\vec{A}(\overrightarrow{\mathrm{r}})$ is the vector potential, e is the charge of the particle, and the integral extends over an arbitrary path between some point, $\overrightarrow{\mathrm{r}}_{o}$ and the field point, $\overrightarrow{\mathrm{r}}_{1}$. Feynman, in his Physics Lectures, ${ }^{13}$ points out the extraordinary effects on the self-interference of charged particles. For instance, placing an ideally infinite solenoid confining the magnetic flux between a screen with two slits and a receiving screen, the recombined wave function at the latter screen is given by

$$
\begin{aligned}
|\Psi(\vec{r})\rangle & =\mid \Psi_{o}>\left\{\operatorname { e x p } \left\{i\left(\frac{2 \pi e}{h c}\right) \int_{\text {pathI }}^{\vec{r}^{\prime}} \overrightarrow{\mathrm{A}} \bullet \mathrm{d} \overrightarrow{\mathrm{s}}\right.\right. \\
& \left.+\exp \left\{i\left(\frac{2 \pi e}{h c}\right) \int_{\text {path II }}^{\overrightarrow{\mathrm{A}}} \overrightarrow{\mathrm{d}} \overrightarrow{\mathrm{s}}\right\}\right\}
\end{aligned}
$$

where, path 1 has been chosen to circle the solenoid from above and path 2 from below. The interference pattern is observed to depend on both the magnetic flux and the difference in path lengths. 
This page intentionally left blank. 


\section{QUANTUM INTERFERENCE AND ENTANGLEMENT OF MASSIVE PARTICLES}

Richard Feynman wrote, "The self-interference of massive particles remains an intriguing phenomenon that has in it the heart of quantum mechanics."' Although a substantial amount of interference effects between correlated photons have been already observed experimentally, there is very little laboratory work on analogous effects for massive particles. Before entering into the main theme in this section, we shall examine some introductory subjects.

\subsection{THE EINSTEIN-PODOLSKY-ROSEN (EPR) PARADOX}

A most counterintuitive aspect of quantum theory is the non-separability of two distant and non-interacting quantum systems that had interacted in the past (recall d'Espagnat theorem). In a celebrated paper ${ }^{14}$ by Einstein, Podolski, and Rosen, the quantum theory was applied to a system of two particles, that are moving in opposite directions with the same value of the linear momentum, after having interacted initially. After moving apart, the particles enter a detector to measure either the momentum or position of each of them independently.

Because the particles had interacted in the past, there will be substantial correlations between the results of measurements on each of the pair. Although it is not possible to simultaneously measure both the position and momentum of one of the particles (thereby violating the uncertainty principle), one could, says EPR, measure either one of these dynamical variables for one particle of the pair and without disturbing the state of the second particle, determine the value of its corresponding dynamical variable with $100 \%$ certainty. How would the remote unprobed particle know which measurement was implemented on the test particle? Can this information be transmitted instantaneously? This is in summary the EPR paradox. In their paper the authors invoked three criteria: ${ }^{\text {is }}$

(a) Reality Criterion "If without in anyway disturbing a system, one can predict with certainty the value of a physical quantity, there exists an element of physical reality corresponding to this quantity." Thus, if we have measured the momentum of particle 1 , then the momentum for particle 2 must be regarded as an element of reality existing separately in particle 2 alone. $^{14}$

(b) Separability Criterion

"Since at the time of measurement the two systems no longer interact, no real change can take place in the second system in consequence of anything that may be done to the first system." Consequently, an element of reality (momentum in particle 2) can 't be instantaneously created by a "spooky" (Einstein's words) action-at-a-distance due to the measurement on particle 1 . 
(c) Completeness Criterion

"Every element of the physical reality must have a counterpart in the physical theory." According to the above criteria. precisely defined elements of reality must exist in the second particle, corresponding to the simultaneous definition of precise values for its momentum and position before we measured its twin on the next galaxy. What happened to the uncertainty principle? Moreover, because the wave function can, at most, specify either momentum or position at a given time with complete precision. one concludes that the wave function does not provide a complete description of all elements of reality existing in the untested particle.

Bohr, who at the time of the 1927 Solvay Congress had survived. victoriously, the attacks on quantum mechanics by Einstein (see Ref. 16 for detailed account), received the EPR onslaught as a bolt from the blue. One is very much tempted to agree with EPR. Surely, consider two identical marbles, except for the color. blue and red, for instance, enclosed in a box. An extragalactic voyager removes one of the marbles without looking, transports it to the next galaxy and then looks to see that he got a red marble, therefore the remaining marble must be, obviously, with $100 \%$ certainty, blue. Moreover, this property was part of its physical reality no matter what the voyager would do to the red marble. Nothing surprising here! The results of a measurement on some aspect of physical reality of an object ought to be explainable by its own elements of reality and those of the experimental apparatus. Whether a photon is reflected or transmitted in a beam splitter does not depend on what happens to its twin far away. Locality and completeness are the basics of the classical description of Nature. Locality prevents instantaneous transmission of physical fields (no argument here) and completeness can be understood as if "every element of physical reality has its marching orders". In an experiment every particle behaves according to its "hidden" instruction sets, i.e., its intrinsic nature. ${ }^{17}$ Nothing is really random, any observed randomness reflecting our lack of knowledge of the hidden archives. The entire scheme is based on common sense and does indeed appear as a correct description of physical reality.

\subsection{BOHR AND QUANTUM MECHANICS HIT BACK}

In the late thirties, Bohr refuted (in some sort of oblique manner, ${ }^{17.18}$ ) the EPR arguments. To better understand Bohr's counter-arguments, we jump to the 1951 arguments of D. Bohm ${ }^{19}$ and follow his line of thought that consists of showing that the EPR paradoxical results, will not be obtained if one renounces the criteria (b) and (c) above that "the world can correctly be analyzed into elements of reality, each of which is a counterpart of a precisely defined mathematical quantity appearing in a complete theory." The assumption that reality is built on a mathematical foundation is a plausible hypothesis based on the extraordinary success of mathematical analysis in mechanics and electrodynamics achieved in the last centuries within the framework of classical, fundamentally deterministic physics. In contrast. quantum theory makes different, but equally plausible, hypothesis in regard to the fundamental nature of matter. The quantum formalism assures that the one-to-one correspondence between mathematical theory and precisely defined "elements of reality. exists only at the classical level of accuracy whereas at the quantum level the mathematical 
description provided by the wave function is not in one-to-one correspondence with the system reality, but only in a statistical correspondence. Yet. Bohm points out the fact that still one asserts that the wave function can. in principle, provide the most complete possible description of the system that is consistent with the actual structure of matter. The justification of the above assertion is that the properties of a given system exist only in an imprecisely defined form as. say potentialities. which are definitely realized in interaction with a classical system such as a measuring device. For instance, consider two noncommuting observables such as the momentum and position of a particle. in general quantum mechanics postulates that neither exists in a precisely defined fashion but that both coexist in a roughly defined form. such that the uncertainty principle is not violated. However, either variable can be more precisely defined at the expense of the degree of definition of the other when in interaction with a measuring device. Consequently. Within the framework of quantum theory, two noncommuting properties are not only incompletely defined and opposing potentialities, but the realization of these potentialities depend considerably on the systems with which the particle interacts. "There are not precisely defined elements of reality in a quantum system." Thus, these remarks contradict the EPR (a) and (c) criteria above. The separability criterion does, in turn, disappear in quantum mechanics in view of d'Espagnat theorem. None of the EPR criteria apply then to quantum mechanics. Have we proved EPR wrong? Probably not, for a complete classical theory may still exist out there. What we have really achieved is to escape the EPR trap by saying: "folks we are using a formalism where all your conditions do not apply". In fact, Bohr's oblique refutal condensed in his remarks to Einstein "your reality differs from mine".

\subsection{THE IRREDUCIBLE RANDOMNESS OF QUANTUM MECHANICS}

In the previous section we have seen the statistical nature of the quantum formalism, but how to calculate probabilities? An excellent account of the subject is the one given by Milburn ${ }^{17}$ that will be summarized here. To develop the subject, Milburn considers the incidence of a beam of light on a beam splitter, the reflected beam propagates to an upward mirror and the transmitted beam to a lower mirror. The reflected beam, from the upper and lower mirrors are made to fall on a second beam splitter so that its two outputs fall in an upper (U) and lower (L) photodetector (see Fig. 1). The path lengths can be adjusted by moving one of the mirrors in such a way that we can arrange this optical system to send all the light output to the $U$ or L detectors or, for instance, split the output equally between the two detectors. The classical wave theory of light easily explains these results. Now let us dim the incident light to the point that only one photon is detected in a given channel width of the analyzer. When the path adjustment is that only the $\mathrm{U}$ detector was active, we are going to see single photons arriving to this detector at random intervals; none to the $L$ detector. Next, a new path adjustment is performed to get half of the beam intensity in each detector. Again, dim the light until only a single count is recorded at either detector in any time interval. We are going to count a single photon at the $L$ detector and none at the $U$ detector and vice versa. Even though we very well know the structure of the incoming light and the nature of the apparatus, which of the two detectors registers one count is "as random as the toss of a coin". We just can't predict the outcome of the measurement. Now switch gears and turn to the theory predicted in the EPR paper; i.e., that the particle carries its own element of reality. 


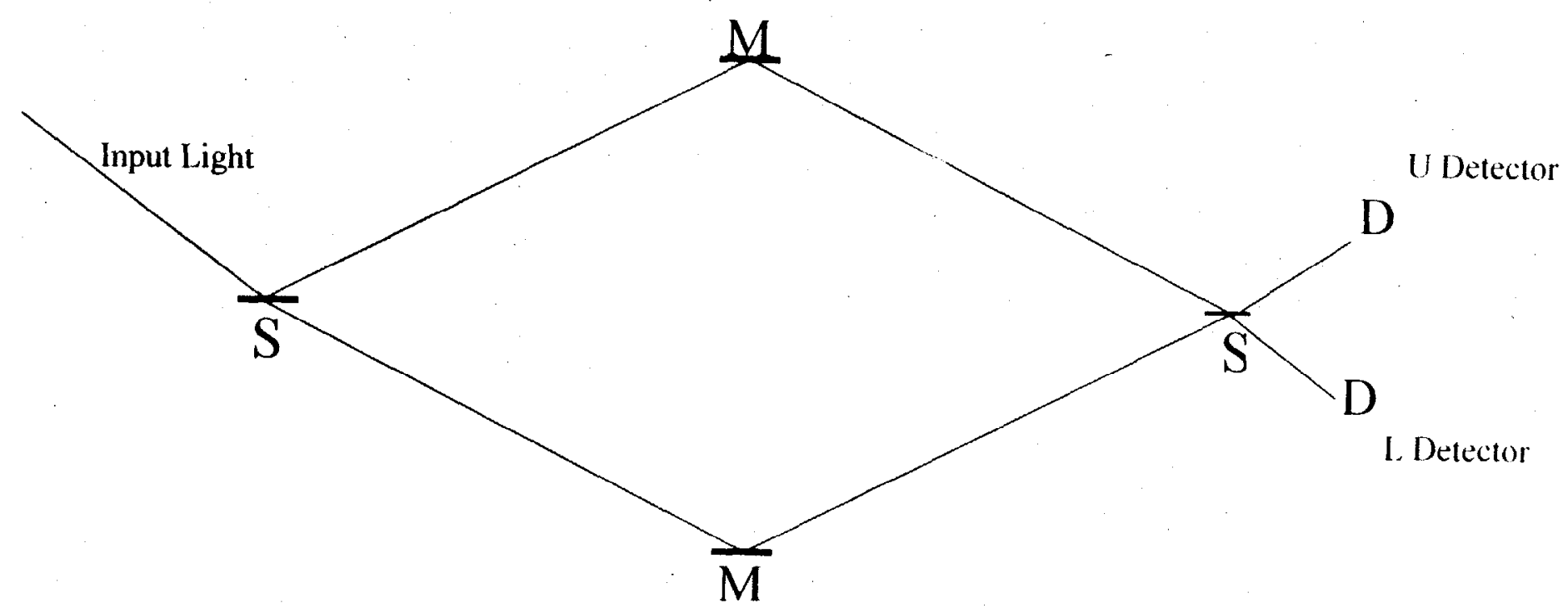

Fig. 1. Milburn ${ }^{17}$ set-up to discuss the wave nature of light.

$S=$ Beam splitter

$\mathrm{M}=$ Reflecting mirror 
Following again Milburn's exposition, assume that photons carry a gene (hidden variable) that determines whether the photon is going to be transmitted or reflected upon impinging on the beam splitter. If its gene is an R-gene, it will be reflected at the first and second beam splitter, thus it will always end up in the upper detector. If it is a T-gene photon, it will be transmitted in both beam splitters and again end up in the $U$ detector regardless of the path adjustment. So much for elements of reality! Going along with the quantum formalism random photon behavior, let's make up the following table of outcomes:

$\begin{array}{lll}\text { Photons reflected at both beam splitters } & \rightarrow & \text { RR } \\ \text { Photons transmitted at both beam splitters } & \rightarrow \text { TT } \\ \text { Photons reflected at the first; transmitted in the second } & \rightarrow \text { RT } \\ \text { Vice Versa } & \rightarrow \text { TR }\end{array}$

$R R$ and TT outcomes lead to detection in the $U$ detector; conversely, the outcomes RT and $T R$ lead to a certain detection in the lower detector. Clearly, Milburn's experiment has to be analyzed in terms of the successive tossing of a coin with one side labeled $R$ and the other labeled $\mathrm{T}$. To determine the chance of a photon being counted at $\mathrm{U}$ or $\mathrm{L}$, one needs a rule for combining probabilities. Amongst the several existing rules perhaps the best know are:

(1) Laplace rule, "In the absence of a prior information, assume equal probabilities for all the possible outcomes". Accordingly, each of the outcomes, RR, TT, RT, and TR, will have a $25 \%$ probability. (2) Bayes rule "If an event can happen in two or more ways, add the probability for each way, considered separately!! Accordingly, a U detection can happen in two ways (RR, TT) and an L detection also in two ways (RT, TR). Hence, we shall have a $50 \%$ probability for both $\mathrm{U}$ and $\mathrm{L}$ detection events. These two rules can be quantified in the following relations:

$$
\begin{aligned}
& P(A+B)=P(A)+P(B)-P(A B) \\
& P(A B)=P(A) P(B / A)=P(B) P(A / B)
\end{aligned}
$$

Both rules lead to probabilities independent on the path adjustment (i.e., the difference between the length of the upper and lower paths). This conclusion is not consistent with the observations. What is missing is the proper combination of randomness with interference. Also, we forgot (momentarily) the fact that in the quantum dictum, probability is not fundamental but derived from the probability amplitude (i.e., the wave function), a complex function depending on two real quantities. It is on the basics of the wave function. $\Psi$, that the probability is calculated as the product $\Psi \Psi^{*}=\Psi_{k}^{2}+\Psi_{1}^{2}$. Hence, the probability is given by the hypotenuse of a right angle triangle (see Ref. 2). Obviously, there is an infinite set of right angle triangles with the same hypotenuse (the absolute direction taken as unimportant). Having said that the amplitudes are the fundamental entities, we return to the beam splitter apparatus in Fig. 1. We shall need two probability amplitudes; one for reflection, and one for transmission. Consequently, two right angle triangles with the same value of the hypotenuse since reflection and transmission are equally likely events. The length of the hypotenuse is all that matters in the case of a single beam splitter since all that we have to describe is a random coin toss. It is when there are more than one beam splitter that we have to face again the issue of combining probabilities in a chain of random events. Not very surprisingly, R. Feynman came to the rescue: Feynman Rule: "if an event can 
happen in two or more indistinguishable ways, the probability amplitude for that event is the algebraic sum of the probability amplitudes for each way, considered separately ${ }^{\circ}{ }^{17}$

Milburn, in Ref. 17, shows how Feynman's rule combines the various probabilities at each beam splitter so that the effects of path adjustments are correctly interpreted. Photons then behave in an intrinsically random way. However, the rules for calculating the probabilities for multiple encounters with a set of beam splitters are quite different from the classical ones. They become important rules for the operation of the quantum computer.

\subsection{ENTANGLEMENT AND BELL'S INEQUALITY}

Quantum entanglement described correlations between the results of local measurements performed on two particles even if the entangled pair is well separated at the time of the measurements. In Section 7.1 we have shown that the quantum mechanical formalism predicts non-local effects, upon a local measurement, something very counterintuitive indeed! The opposite view expounded in Section 10.1 is that it can't exist spooky action ata-distance and that each particle carries its own "element of reality" (hidden variables). The Bohr-Bohm refutal and the examples in Section 10.2, where the irreducible randomness of quantum mechanics was discussed, indicated the problematics of local, hidden variable theories but did not provide a "measurable" test. It was the Irish Physicists, John S. Bell, who provided such a test ${ }^{20}$ in a short and fundamental paper. We follow here a special formulation of this test; the so-called Bell's inequality provided in Ref. 4. Consider iwo photons moving in opposite directions and having the two circular polarized states, $\mathrm{m}_{1}(1)$ and $\mathrm{m}_{1}(2)$, for the subspace $\mathrm{H}(1)$ and $\mathrm{m}_{2}(1)$ and $\mathrm{m}_{2}(2)$ for the $H(2)$ subspace. It can be shown that although the photon carries spin 1 (boson type), the operator associated to the states defined above is the spin operator, $\hat{\sigma}_{z}$. Thus, we can carry over the formalism developed in

Section 7 and the following. The polarization operator associated with the polarization states above is the measurement operator, given by

$$
\hat{\mathrm{A}}(v)=\hat{\sigma}_{x} \sin \theta(v)+\hat{\sigma}_{z} \cos \theta(v)
$$

where $\theta(v)$ is the angle setting of the polarizer. The corresponding expectation value vectors, $\overrightarrow{\mathrm{A}}(v)$, are calculated in Appendix A:

$$
\begin{aligned}
& \overrightarrow{\mathrm{A}}(1)=2[\sin \theta(1), 0, \cos \theta(1)] \\
& \overrightarrow{\mathrm{A}}(2)=2[\sin \theta(2), 0, \cos \theta(2)] .
\end{aligned}
$$

Now imagine that on both sides of the photon source there have been placed two polarizers, $A$ and $B$. We perform two measurements. In the first, the polarizer $A$ is set at the angle $\theta_{1}$, and $B$ at $\theta_{2}$. In the second, these angles have been changed to $\theta_{3}$ and $\theta_{4}$, respectively. After the measurements, one can obtain the cross correlations, $C_{A A}\left(\theta_{1}, \theta_{2}\right), C_{A A}\left(\theta_{2}, \theta_{3}\right)$, 
$C_{A A}\left(\theta_{3}, \theta_{4}\right)$. and $C_{A A}\left(\theta_{4}, \theta_{1}\right)$. Bell's inequality then establishes that the following condition must be satisfied within the framework of "local realism"

$$
S_{o}=\left|C_{A H}\left(\theta_{1} \theta_{2}\right)+C_{A H}\left(\theta_{2} \theta_{3}\right)+C_{. t H}\left(\theta_{3} \theta_{+}\right)-C_{A H}\left(\theta_{4} \theta_{1}\right)\right| \leq 2 .
$$

To see how the quantum mechanical correlations. $C_{A A}$, behave with regard to Bell's. inequality (10.4) we use Eq. (7.35) (see also Appendix E) to obtain

$$
C_{A, 1}\left(\theta_{1} \theta_{2}\right)=-\cos \left(\theta_{2}-\theta_{1}\right) \text {. }
$$

Now choosing the angles in the sequence, $\theta_{2}-\theta_{1}=\theta_{3}-\theta_{2}=\theta_{4}-\theta_{3}=\varphi$, and $\theta_{4}-\theta_{1}=3$ ( 0 . Bell's inequality becomes

$$
S_{w}=|3 \cos \Psi-\cos 3 \Psi| \leq 2
$$

that is obviously violated for instance if $\varphi=\frac{\pi}{4}$, among other values. Clauser. Horne. Shimony, and Holt ${ }^{21}$ arrived at stronger inequalities. Many experimental tests have been performed of Bell's; inequality (see the surveys by Clauser and Shimony, ${ }^{22}$ Pipkin. ${ }^{23}$ and Horne and Selleri. ${ }^{24}$ All measurements confirm the predictions of quantum mechanics. However, Weihs, et al. ${ }^{25}$ point out the fact that all this amount of evidence still does not rule out a local realistic explanation completely because of three loopholes in the experiment. (1) Due to low efficiency of detection, only a small fraction of the pairs emitted by the source are actually detected. Consequently, one has to assume that this detected subset is a fair representation of all emitted pairs. (2) "The settings of the measuring systems are made sufficiently in advance to reach some mutual rapport by exchange of signals with velocity less than or equal to that of light... it is then crucial to change settings during the flight of the particles," Bell. ${ }^{26}$ (3) The assumption of locality in the derivation of Bell's inequality requires that the two observers be space-like separated.

The first measurement where the experimental settings were switched was due to the Aspect et al. ${ }^{27}$ Weihs et al. ${ }^{25}$ performed a Bell's inequality test under strict Einstein locality conditions by switching at random the photons polarization angle and placing the measuring systems separated by $400 \mathrm{~m}$ across the Innsbruck University campus.

\subsection{QUANTUM INTERFERENCE OF MASSIVE PARTICLES}

In contrast with the substantial amount of experiments to study interference effects between correlated photons, the laboratory realizations of analogous effects for massive particles are scarce. The interest in the experimentation of interference effects for massive particles lies in that the attributes of charge and quantum statistics (fermions as opposed to the bosonic statistics for photons) when combined with the $A B$ effect (see Section 9) would manifest three distinct types of quantum interference: ${ }^{28}$ (1) interference dependent upon optical path length difference resulting from wavelike propagation of particles; (2) interference dependent 
upon confined magnetic flux resulting from particle charge and spatial topology. and (i) interference dependent upon quantum statistics resulting from particle indistinguishability under exchange. Two types of experiments will be reviewed: particle self-interference and quantum interference with correlated massive particles.

\subsection{PARTICLE SELF-INTERFERENCE EXPERIMENTS}

Particle self-interference experiments are used as a tool to test "the fundamental principles of quantum mechanics with massive particles on a macroscopic space-timed scale. ${ }^{.15}$ Selfinterference experiments have been performed with neutrons and electrons.

(a) Neutron Self-Interference

The neutron interferometer consists of a perfect silicon crystal cut in the form of three plane-parallel plates on a common base. The incident neutron beam splits into two coherent beams that are superposed by the middle plate in the third plate acting as the analyzer of the resulting interference pattern. ${ }^{15}$ Additional parallel plates can be used as phase shifters to adjust the path difference between the two neutron beams. Rauch and Summhammer ${ }^{30}$ performed neutron interferometry measurements introducing a neutron absorber in one of the neutron beams. Badurek et al. ${ }^{31}$ introduced resonance spin-flip coils in both neutron beams to investigate the nature of wave-particle duality. Extensive description of these experiments are given in Ref. 15. Phase shifting of neutron beams by the $A B$ effect (see Section 9) was first suggested by Zeilinger ${ }^{32}$ and Anandan. ${ }^{33}$ Several AB-based measurements were performed by Allman et al. ${ }^{34}$ using unpolarized neutron beams and by Lee et al. ${ }^{35}$ with longitudinally polarized neutrons.

(b) Electron Self-Interference

Tests of the wave-particle duality using coherent, low-intensity electron beams have been performed by Tonomura et al. ${ }^{36}$ In analogy to the standard double-slit experiment. Tonomura used a special electron gun emitting coherent wave fronts. By blocking their central parts, one recreates a double slit. ${ }^{15}$

\subsection{QUANTUM INTERFERENCE EXPERIMENTS WITH CORRELATED MASSIVE PARTICLES}

Quantum interference experiments with correlated beams of electrons interacting with confined magnetic fields (AB-effect) have been extensively discussed by Silverman. ${ }^{28}$ However, as pointed out by Silverman himself, the proposed measurements would need the use of sources of correlated electrons, not yet available. Ghose ${ }^{15}$ describes a test of an EPRlike situation using the decay of the vector meson $\Phi$, into a kaon anti-kaon split. This test has been studied by several authors. The main difficulty of observing its predictions lies in the short lifetime of the kaon products $\left(\sim 10^{-10} \mathrm{~S}\right)$. 


\subsection{NUCLEAR REACTIONS AS SOURCES OF CORRELATED PARTICLES}

In view of the availability both present (High Flux Reactor) and future (Spallation Source) of neutron with energies from subthermal up to a few $\mathrm{MeV}$. it appears of interest to look at neutron reactions as sources of correlated massive particles. Two main requirements arise from the analysis of the previous sections: (1) superselection rules. and (2) the particles are correlated either because of the reaction mechanism itself and/or because of the overlapping of their wave functions (d'Espagnat theorem). Superselection rules will eliminate those nuclear reaction product pairs of different charge and quantum statistics of their components.

In regard to the second requirement, direct reactions involving the formation of an excited nucleus of low mass number appears more promising that reactions in heavier nuclei with the formation of Bohr compound nucleus. This is because the de-excitation of the reaction product low mass number does not have as many decay channels as for instance the $(\mathrm{U}-235+n)$ compound nucleus.

A possible exception could be subthreshold fission processes where "privileged" exit channels appear by the matching of levels in the first well of the fission barrier with the much less populated level set in the second well of the barrier. In general though. there can't be too much hope that neutrons coming either from the same or different fission products could be correlated. A nuclear reaction that appears promising is the interaction of slow neutrons with helium 3 to produce protons and tritons:

$$
{ }_{2} \mathrm{H}_{c}^{3}+{ }_{{ }_{0}} \mathrm{n}^{1}=\left({ }_{2} \mathrm{H}_{v}^{4}\right)^{*}={ }_{1} \mathrm{P}^{1}+{ }_{1} \mathrm{~T}^{3} ;\left[\mathrm{E}_{\mathrm{p}}=.573 \mathrm{Mev}, \mathrm{E}_{\mathrm{\tau}}=.191 \mathrm{Mev}\right]
$$

This reaction goes through the formation of an unstable $\mathrm{He}-4$ nucleus that decays into one proton and a triton flying in opposite directions with the same momentum. Both particles have the same charge and are both fermions (half-integer spin). However, they have different masses and therefore they can be "separated" by the gravitational field. We shall assume that, in view of the smallness of the interaction, all the conditions to pass the superselection rule requirement are being satisfied. Since the pair of reaction products are highly correlated in momentum space, one expects also a high level of correlation in their location at the moment of production (not to be seen as a violation of the uncertainty principle). There is then a good chance that either by the reaction mechanism or both by this mechanism and wave function overlapping the two particles come up correlated. A detailed study of this reaction as a possible source of entangled particles will be presented in a separate report. 
This page intentionally left blank. 


\section{QUANTUM COMPUTATION AND INFORMATION PROCESSING}

It was R. Feynman, who in 1982 suggested $^{37}$ that individual quantum systems could be used for computations. The first description of a universal quantum computer was given by $D$. Deutsch $^{38}$ in 1985. At the rate of which lithographic techniques advance presently, there will be a point where logic gates will be made of a few atoms. At this microscale limit quantum effects will play a role so that classical devices may be either supplemented or replaced by quantum gadgets. Lengthy and ever much exciting reviews of the fields of Quantum Computation (QC) and Quantum Intormation Processing (QIP) are given in the books of G. Milburn, "The Feynman Processor," and M. Brooks," "Quantum Computing and Communications." For late developments, see the recent article by Birnbaum and Williams ${ }^{40}$ in Physics Today. In a nutshell (see Ref. 39) a quantum computer has to have at least four components: Qubits, a set of logical gates, an initialization (CLEAR) operation, and a readout mechanism. In this report, we shall only worry about qubits that are the most fundamental components.

\subsection{QUBITS AND QUANTUM SOFTWARE}

Present computers collect and handle information using what is called a "bit," i.e., an entity that can take on one of two values. Arbitrarily, bits are labeled by the two digits, 0 , and 1 ("on" and "off"). The values taken by the bit are encoded by some electric or magnetic means with the condition that these values are sufficiently separated in their parameter space for information reliability.

Now let's see how we could store information in a quantum mechanical way. Consider an atom and its discrete set of electronic energy level. One could label the fundamental energy level " 0 " and the next one "l" thus similarly to a classical bit, information would then be encoded in the atom. Clearly, one can also encode information in photons by using polarization states or by encoding in terms of electron spin components. However, (recall Section 3) the atom can enter into a superposition of states: $\left.C_{1}\left|0>+C_{2}\right| 1\right\rangle$, in terms of the kets, representing the two states. From the superposition principle comes the first insight into the potentiality of quantum computing. Now the atom can represent " 0 " and " 1 " besides " 0 " or " 1 ". We now have a "qubit".

Imagine that we have available to us a register of L-qubits. Due to quantum superposition. the register can now store $2^{\mathrm{L}}$ different numbers at once on which one can perform operations simultaneously. Thus, one can implement a massive parallel computation in a single piece of quantum hardware. To accomplish the same task, a classical computer will have to repeat the calculation $2^{\mathrm{L}}$ times. Following Feynman's suggestion, ${ }^{37}$ the first proposed application was the simulation of quantum systems. Consider a quantum system with $\mathrm{N}$ degrees of freedom. The corresponding Hilbert space will have $2^{\mathrm{N}}$ dimensionality. According to the evolution equation (2.44), one would have to evolve in time matrices of dimension $2^{\mathrm{N}}$. Following the time evolution of a quantum system containing 40 spins is an impossible task 
for any classical computer today and in the far future. In 1996. Lloyd ${ }^{41}$ showed Feynman conjecture to be correct.

In the realm of quantum software two fundamental contributions are Shor ${ }^{42}$ factorization algorithm and Grover's search algorithm. In 1993, Shor ${ }^{+2}$ (AT\&T Bell Labs) developed a quantum algorithm that can factor large integers in poly nomial time (i.e.. the time it takes to execute the algorithm increases no faster than a polynor'ial function of the input size) in this case the problem belongs to a P-class. Problems outsid this class are called hard problems. The factorization problem has been qualified as "hard" ior classical computers. For example. for a classical computer to factorize a one thousand digits number would take more than the estimated age of the universe. In 1997, Grover ${ }^{43}$ described a quantum algorithm to perform searches in a random database. For a record of $\mathrm{N}$ items, classical algorithms require an

average of $\frac{N}{2}$ steps. Grover's algorithm performs the same search in $\sqrt{\mathrm{N}}$ steps.

\subsection{ENTANGLEMENT}

In Section 7 of this report, we have studied the joint state of two quantum systems and their correlation function. We showed how a local interaction on one of the particle pair induces a change in the quantum state of its partner. Consider now that we have two qubits and that we like to encode two bits $\mid 0>$ and $\mid 1>$. One can encode one bit onto each qubit separately, for instance $\left|0>_{1}\right| 0>_{2},\left|0>_{1}\right| 1>_{2},\left|1>_{1}\right| 0>_{2}$ and $\left|1>_{1}\right| 1>_{2}$, or one can encode the information onto an entangled state of the two qubits so that none of the qubits carries any definite information on its own. A much used entanglement choice is based on the so-called Bell states:

$$
\begin{aligned}
& \mid \Psi(+)>=\frac{1}{\sqrt{2}}\left(10>_{1} 11>_{2}+11>_{1} 10>_{2}\right) \\
& \mid \Psi(-)>=\frac{1}{\sqrt{2}}\left(10>_{1} 11>_{2}-11>_{1} 10>_{2}\right) \\
& \mid \Phi(+)>=\frac{1}{\sqrt{2}}\left(10>_{1} 10>_{2}+11>_{1} 11>_{2}\right) \\
& \mid \Phi(-)>=\frac{1}{\sqrt{2}}\left(10>_{1} 10>_{2}-11>_{1} 11>_{2}\right)
\end{aligned}
$$

It is possible to derive any one of the Bell states from any other be performing a unitary transformation (see Section 2) on one of the two qubits (there are four possible unitary transformation with this property: identity, phase shift, bit flipping, and shift and bit flipping. Note that due to the non-locality property of entangled states unitary transformations are "seen" by both entangled particles. Entanglement allows for various operations unthinkable in classical physics: dense coding, cryptography, and teleportation. the paradigms of QIP. 


\subsection{DENSE CODING}

Observers A (Alice) and B (Bob) are supplied (from an appropriate source) with two photons entangled in one of the four Bell states above. Alice applied on her photon a unitary transformation transferring the pair into one out of the four possible Bell states. Afterwards. Alice sends her photon to Bob which can be detected after he gets hold of the total system. Since there are four possible outcomes of Bob's measurement, Alice has sent twice as much information as can be sent classically with a two-state particle (four alternatives instead of two). This experiment was first implemented by Zeilinger group at Innsbruck. ${ }^{44}$

\subsection{QUANTUM CRYPTOGRAPHY}

"Cryptography consists of hiding information in a string of bits that are meaningless to any unauthorized party." ${ }^{39}$ Cryptography algorithms are used to combine a message with some additional information known as the "key". The key consists of a set of specific parameters introduced into encrypting and decrypting algorithms. The transmission of the key to interested parties must be protected from eavesdropping, a difficult task when classical communication channels are used. If this security cannot be implemented, the next best thing is to make sure that eavesdropping can be detected. This can be implemented by using a quantum channel for the transmission of the key. In one version, the two-particle entangled state (EPR-state studied in Section 10.3) is the basis for the implementation of the quantum channel. It works as follows: ${ }^{4}$ Alice picks three discrete angles, $\theta_{1}=0, \theta_{3}=\frac{\pi}{4} ; \theta_{5}=\frac{\pi}{2}$. In turn Bob picks $\theta_{2}=\frac{\pi}{4} ; \theta_{4}=\frac{\pi}{2}$ and $\theta_{6}=\frac{3 \pi}{4}$. The source sends entangled photon pairs, one member of each pair goes to Alice and the other to Bob. Both observers are randomly changing their respective allowed angle settings. The detected events are registered in local protocols, and Alice and Bob transmit their respective sets of angles in a public channel. The set of results obtained are (arbitrarily) split into two subsets: (1) same orientation $\left.\left[\theta_{3}, \theta_{2}\right),\left(\theta_{5}, \theta_{4}\right)\right]$, and six different orientations, $\left[\left(\theta_{5}, \theta_{2}\right),\left(\theta_{1}, \theta_{2}\right),\left(\theta_{5}, \theta_{6}\right),\left(\theta_{1}, \theta_{6}\right)\right]$. The first set is kept as the key; the second set is transmitted through a classical channel so that both observers can check that Bell's inequality (10.4) is violated. In fact, for the set picked by Alice and Bob, Eqs. (7.35) and (10.4), we have

$$
\mathrm{S}_{u}=\left|3 \cos \left(\frac{\pi}{4}\right)-\cos \left(3 \frac{\pi}{4}\right)\right|=2 \sqrt{2}>2
$$

If one finds that $S_{o} \neq 2 \sqrt{2}$, the quantum channel has been messed with. ${ }^{4}$ This scheme for the quantum transmission of a key has been implemented by Ekert. ${ }^{45}$ 


\subsection{QUANTUM TELEPORTATION}

Teleportation consist of the transmission of the state of a photon. For instance, Alice has to teleport an unknown state $\Psi=a|0>+b| 1>$ ) to Bob; let's call this photon (1) (qubit (1)). An entangled pair of photons $(2,3)$ is now shared by both observers. At a time, say $t_{1}$, Alice gets the photon (2) and Bob the photon (3). Thus, Alice now has a three-node entangled state (1)-(2) (3). At time, $t_{2}$, Alice performs a measurement on her two photons (1) and (2), with respect to the four Bell states. Two events follow: (a) Alice's measurement has led to one of the four Bell states that after the collapse of the state, provides two classical bits of information, and (b) due to non-local interactions, Bob's photon (3) has changed to a state congruent with the outcome of Alice's measurement. Now, if Alice phones the two classical bits from her measurement to Bob, he recognizes which unitary transformation was used by Alice. All we have to do now is to apply the inverse unitary transformation to his photon (3), to acquire the state of photon (1). Thus by applying this transformation, Bob has received (1) without measuring it or Alice actually transmitting it. Reference 4 presents the rigorous theory of the teleportation scheme. See also Bennet et al. ${ }^{46}$ Experimental confirmation has been provided by Bouwmeester et al. ${ }^{47}$

\subsection{QUANTUM COMPUTER HARDWARE}

Both Refs. 17 and 39 contain a detailed study of the present ideas about the hardware to implement logic gates and the quantum software. The main contenders for the job are up to now:

(a) Trapped Ions Ignacio Cirac at the University of Castilla La Mancha and Peter Zoller at the University of Innsbruck ${ }^{48}$ suggested storing a collection of ions in an electrostatic trap with each qubit (one per ion) formed by a pair of internal states of the ion. Because of the ion confinement, it is feasible to entangle the motion of all the ions and all their respective electronic states. Stean $e^{49}$ has implemented a control-not gate using one trapped ion. One expects that ion trap quantum computers can be built. Although this type of system does not have a fundamental scaling limit, decoherence effects will act very destructively beyond more than 10 ions in the trap.

(b) Nuclear Magnetic Resonance (NMR)

In NMR the two spin states of an atomic nucleus immersed in a magnetic field serve as the basis of a qubit. Since NMR can distinguish among the atoms of a molecule, it can be used as a quantum computer whereby each nucleus provides a single qubit. Because of the weakness of the NMR signal from a single molecule, it is necessary to amplify the signal by using a large number of identical copies (not too difficult to implement in view of Arogrado's number). The problem is that it is practically impossible that all the molecules start from the same initial state (clearing the computer) so different copies will perform different calculations. In fact, one ends up with a mixture of final states. Several solutions to "distill" the desired state from the mixture have been developed. ${ }^{50}$ Several implementations of NMR computers have been realized. ${ }^{51}$ 
(c) Cavity Quantum Electrodynamics

In cavity quantum electrodynamics atoms are held in an optical cavity. Lnder appropriate conditions, the light can be used to couple and control the quantum states of the cavity "trapped" atoms. The dynamical theory of an atom interacting with quantized cavity fields have been studies by Yao.

(d) Quantum Dots

Quantum dots are regions on the nano meter scale where electrons remain confined and their energies quantized as a consequence of confinement. Since quantum dots do mimic the electronic levels of actual atoms, they can be used. in principle. to encode and store quantum information, as well as a new source of single photons. Also, having interacting quantum dots will provide a basis for the implementation of quantum logic gates. The dynamics of nanostructures has been extensively studied. ${ }^{4.53 .54}$ 
This page intentionally left blank. 


\section{PROBLEMS AND RESEARCH ISSUES}

It is always a bit dangerous to jump on the bandwagon of attractive new technologies without first looking at their "ugly" side of the face. For instance, look what happened to the "AllOptical" computer. ${ }^{55}$ For the following discussion it is convenient to recall Moore's Laws: Gordon Moore (Intel Corp.) quantified the steady increase of gate density bringing up the first Moore's Law, "The number of transistors built into a chip increases exponentially with time," and Moore's second law, "The cost of building a chip factory also increases exponentially."

Moore's first law has been used as an argument for the development of quantum computers. It goes like this, more and more transistors will create a situation whereby transistor components will get smaller, at this point quantum effects will appear so let's go quantum anyway. However, in view of Moore's second law, miniaturization is slowing down, not for physical but for economic reasons. The semi-conductor industry may go away form miniaturization and enter other ways of expansion, like for instance, 3-D integration since what counts is component density rather than size. ${ }^{56}$ This new direction may lead the semiconductor industry into new architectures that could make the quantum computer look like an expensive toy.

In the event that one goes quantum, there are three main interrelated serious problems that demand active research: unitarity, decoherence, and errors.

(a) Unitarity

All operations in a quantum computer are performed on the basis of unitary transformations (assuming evolution according to the Schrödinger equation). Qubits evolve in the form of unitary (no damping) transformations (see Section 2, Eq. 2.44 and Section (1). This means that while computing the computer must be described and kept on a closed system since any observation on its working will collapse the wave functions of the entangled qubits. Hence, interaction with the external world (to get the results, for instance) must be realized after a flag indicated the end of the computation. The problem is to keep external disturbances from entering the system.

(b) Decoherence

In Section 8 we have briefly discussed the subject of decoherence. Quantum decoherence is one of the major roadblocks to quantum computation. The problem is that the computation process occurs in terms of the parallel evolution of entangled qubits along different pathways carrying definite phase relations. At the end they must interfere to produce a given result that can be prevented to arise due to the loss of entanglement by decoherence. ${ }^{57}$

(c) Errors

Two kinds of errors appear in the dynamics of a quantum computer; classical bit-flip errors and superposition destroying errors due to decoherence. However, as pointed out by Landauer ${ }^{59}$ there are other sources of errors besides decoherence. They arise from the 
physical implementation of the quantum mechanical operator associated with the unitary transformation you want in your computer. Fabrication defects will make these implementations only approximately correct. Thus, every single processor (like gates) in the device will be somewhat off in a systematic manner from the ideal situation. 


\section{FUTURE RESEARCH}

There are, for now, two methods to prevent decoherence.

(a) Control of Quantum Systems

Similar in many aspects to classical control, the quantum version still consists of the minimization of some functional that may be constrained by some demands on the system. There ends the similarity since now the basis of the system is the quantum Hamiltonian. For closed systems the model describing the gadget to be controlled is the quantum Liouville, equation (2.43). When open to the environment (see Section 8), one must include in the Hamiltonian the effect of the environment. As a result for the reduced, traced upon system the time evolution is not unitary any more. For instance, the time correlation of the system is damped; i.e., it decays exponentially with time and reversibility is lost. ${ }^{4}$ The task of the control algorithm is to determine the kind of external fields that can either prevent the environment effects or impose a given output demand on the system environment complex.

Much of the work done on quantum control ${ }^{58,59.60 .61 .62}$ is in the class of open-loop control; that is, there is no feedback signal. In this instance, changes in the system parameters will disrupt control. The problem with the concept of feedback (closed loop control) is that one has to be mindful to the fact that the feedback loop would not behave as another interaction with the external world, which it really is. The solution of this problem would allow the use of uncertain dynamics to account for the effect of the fabrication flaws mentioned in Section 12.

(b) Quantum Error Correction

By the end of 1996, new principles were discovered that enabled quantum computers to be protected from errors, classical bit-flip as well as decoherence errors. The idea ${ }^{17}$ is based on the use of redundancy by using strings of bits to encode a single " 1 " or " 0 ". This encoding is so implemented that an error in a single qubit still will allow the original qubit to be inferred and restored. ${ }^{39}$ Research on this subject is very much active. $^{63,64,65}$ 
This page intentionally left blank. 


\section{OVERVIEW}

It is now apparent that quantum physics could propel computing beyond the silicondominated era (see Ref 40 for a most interesting overview of the future of computing). In a broad sense. three fields of research and applications seem to arise in the area of Quantum. Information Processing.

(a) Quantum Switches

According to predictions from the Semiconductor Industry Association. ${ }^{+1}$ in 2010 the individual transistors will be turned on/off by the addition or removal of a few electrons. The robustness in distinguishing " 0 " from " 1 " will be compromised. This would be a good time to develop quantum switches!

(b) Secure Communications

As it was discussed in Section 11, quantum communication provides a safe method of transferring information, with the possibility of applying statistical tests to verify if any eavesdropping has been attempted.

(c) Quantum Logic Applications

Systems handling only a few qubits can presently be build, which will be an incentive to perform quantum simulations of interest to the chemical and pharmaceutical industry. Other industrial applications such as metrology (improved precision atomic clocks. quantum gyroscopes. navigational aids) can be implemented with small ( 10 qubits) systems.

(d) Full-Blown Quantum Logic Computers Machines with hundreds of entangled qubits. using quantum logic because of their reversibility and inherently parallel features, represent a leap far beyond what classical computers can offer. ${ }^{40}$ However; barring unexpected theoretical and technical developments, the general belief for the implementation of such a machine sets the date between 2025 and never. 
This page intentionally left blank. 


\section{SHOULD ORNL BE INVOLVED IN QUANTUM INFORMATION PROCESSING RESEARCH?}

Intermediate between Lniversity and Industry lies ORNL. Universities have done most of the basic theoretical and experimental research in QIP. For them the issue of participation or not depends on getting, or not getting. appropriate funding from the National Science Foundation (or other government sources of funds). In regard to Industry. the motivation is to show benefits. The amount of resources to be committed depends on an estimation of the siz if the future market for QIP. R. S. Williams. Director of Basic Research for Hewlett Pachard Laboratories, in Ref. 39, provides a fascinating account on how to figure out the amount of company funds to be assigned to basic research in QIP: ten million dollars year. This figure though may have been obtained at the time (1999) on the basis of a somewhat pessimistic view of the QIP field since presently one can observe a more intense desire to work in this area by the Hewlett Packard Company. ${ }^{10}$

A possible approach for ORNL is not to consider the construction of the full-blown quantum computer right away. Rather than this, one should consider those areas of research. as for instance quantum communication. either close to industrialization or arising interest for commercialization by the Industry. However, there has to be a basic research component to observe and measure quantum phenomena, as for instance the work presently performed at the Instrumentation and Controls Division with entangled photons and molecular computers. Incidentally. Los Alamos National Laboratory (LANL), one of the most far looking ahead of the national laboratories, is already heavily engaged in teleportation and quantum cryptography. The next component of the research program is two folds: move towards experimentation to control the observed quantum phenomena and look for new technologies and materials to implement nano-circuitry escaping from Moore's second law. ORNL has indeed an excellent "know-how" in both areas.

The amount of resources to be engaged in this kind of "risky" subject is certainly depending on the funding extracted from NASA, DOE, and DARPA and from the extense of cooperative agreements with Industry. Participation in QIP is a calculated risk, balanced by the perception that one has to sustain the economic benefits to the U. S. economy due to advanced computation when the second Moore's law stops miniaturization. Finally. ORNL is full of bright, young, computer wizards. ' 't's challenge them! 
This page intentionally left blank. 


\section{REFLECTIONS ON QLANTUM MECHANICS}

Quantum mechanics is an amazingly successful formalism that describes nuclear atomic and high energy particle phenomena with great accuracy. However. almost from its inception. Einstein ${ }^{14}$ considered quantum mechanics to be an incomplete description of the physical reality. The delayed impact of Einstein's remark was felt when very precise experiments of photons, neutrons, and single atoms in electromagnetic cavities were profusely reported in the literature and in special when the teleportation of entangled quantum states were actuall! implemented. Although very counterintuitive, entanglement arises directly from the quantum formalism (Dirac's superposition principle). The real problem though is the act of measurement involving the interaction between macro and quantum objects. To describe measurements quantum mechanically, von Neumnn "dogmatized" his axiomatic measurement theory (see Section 5) and started a long and not yet clarified polemic of when (and if) the time evolution described by the unitary transformation actually leads to the collapse of the wave function. Consequently, there is not a complete and unambiguous description of one of our most fundamental physical theories' (Einstein does indeed cast a long shadow). Nevertheless, the quantum formalism has not been contradicted yet by any measurement. Its mathematical formalism predicts entanglement and non-locality as it has been experimentally confirmed. In view of so many clear-cut successes, one may ask whether the polemic on the collapse of the wave function or on the limits between the quantum and classical worlds is simply a philosophical polemic. In the words of Wigner ${ }^{66}$. "We are facing here the perennial question whether we physicists do not go beyond our competence when searching for philosophical truth." 
This page intentionally left blank. 


\section{REFERENCES}

1. A. Galindo and P. Pascual. "Mecanica Cuantica," Eudema Publications (Lniversidad Complutense) 1989.

2. L. L. Schiff. Quantum Mechanics. McGraw-Hill Book Company. New York. 1949

3. E. P. Wigner. Interpretation of Quantum Mechanics in Quantum Theorl and Measurement, Princeton Series in Physics, ed. J. A. Wheeler and W. H. Zurek. 1983

4. G. Mahler and V. A. Weberruss, Quantum Networks: Dynamics of (Ipen Nanostructures, Springer-Verlag, Berlin, New York, 1998.

5. P. A. M. Dirac, The Principles of Quantum Mechanics, Oxford at the Clarendon Press, $3^{\text {rd }}$ ed.. 1947.

6. M. Gell-Mann and A. Pais, Physics Review. 97, p. 1387, 1955.

7. E. P. Wigner, "The Problem of Measurement," local citation reference 3. p. 324.

8. F. London and E. Bauer, "The Theory of Observation in Quantum Mechanics," local citation reference 3 , p. 217 .

9. J. von Neumann, "The Measuring Process," local citation reference 3. p. 549.

10. B. d'Espagnat, ConceptualFoundation of Quantum Mechanics, Benjamin-Cummings. Menlo Park, 1971.

11. D. Givlini, E. Joos, C. Kiefler, J. Kupsh, I. O. Stamatescu, and H. D. Zeh. Decoherence and the Appearance of a Classical World in Quantum Theory, SpringerVerlag, Berlin, New York, 1996.

12. Y. Aharonov and D. Bohm, Phys. Rev., 115, p. 485, 1959.

13. R. P. Feynman, R. B. Leighton, and M. Sands, The Feynman Lectures on Physics, Vol. 3, Addison-Wesley, Reading, MA (1965).

14. A. Einstein, B. Podolski, and N. Rosen, "Can Quantum-Mechanical Description of Reality be Considered Complete?" Physics Review, 47, p. 777 (1935).

15. P. Ghose, "Testing Quantum Mechanics on New Ground," Cambridge University Press, p. 141 (1999).

16. J. A. Wheeler and W. H. Zurek. Editors, "Quantum Theory and Measurement." Princeton Series in Physics. A detailed description of the Bohr-Einstein dialog is given (1983). 
17. G. J. Milburn, Quantum Entanglement and the Computer Revolution: The Fernmun Processor, Perseus Books. Reading. MA. 1998.

18. N. Bohr. "Discussions with Einstein on Epistemological Problems in Atomic Physics." in On Atomic Physics and Human Knowledge Vol. II: Essays 1932-1957. Ox Bow Press, Woodbridge, CO (1987).

19. D. Bohm. "The Paradox of Einstein, Rosen. and Podolski." local citation 3. p. 356.

20. J. S. Bell, "On the Einstein, Rosen, Podolski Paradox," Physics, 1. 195 (1964).

21. J. F. Clauser. M. A. Horne: A. Shimong, and R. A. Holt. Phy: Re'v Let. 23. 880 (1969).

22. J. F. Clauser and A. Shimony, Rep. Prog. Phys., 41. 1981 (1978).

23. F. M. Pipkin. Advances in Atomic and Molecular Physics, ed. Bates and Bederson. Academic Press, New York (1978).

24. D. Home and F. Selleri, Nuoro Cimento, 14, No. 9, 1 (1991).

25. G. Weihs, T. Jennewein, C. Siman, H. Weinfurter, and A. Zeilinger, Phys. Rev. Let., 81 No. 23, 5039 (1998).

26. J. S. Bell, J. Phys. (Paris), Colloq. Suppl. 3, C2, 41 (1981).

27. A. Aspect. J. Dalibard, and G. Roger, Phys. Rev. Let., 491804 (1982).

28. M. P. Silverman, Am. J. Physics, 61, 6 (1993).

29. K. S. Krane, Introductory Nuclear Physics, John Wiley \& Sons, New York. p. 465 (1987).

30. H. Rauch and J. Summhamer, Phys. Let., A 104, 44 (1984).

31. G. Badurek, H. Ranch, and D. Tuppinger, Phys. Rev.. A34, 2600 (1986).

32. A. Zeilinger, Fundamental Aspects of Quantum Theory, ed. Sorini and Frigerio. Plenon, New York (1985).

33. J. Anandan, Phys. Let., A 138, 347 (1989).

34. B. E Allman et al., Phys. Rev., A48, 1799 (1993), 
35. W. T. Lee, O. Motrunich, B. E. Allman, and S. A. Werner, Phys. Rev. Let., 80, No. 15,3165 (1998).

36. A. Tonomura et al., Am. J. of Physics, 57117 (1989).

37. R. P. Feynman, "Simulating Physics with Computers," Int. J. Theoretical Physics, 21, 467 (1962).

38. D. Deutsch, Proc. Royal Soc. of London, A400, 97 (1985).

39. M. Brooks, "Quantum Computing and Communication," Springler-Verlag, London (1999).

40. J. Birnbaum and S. Williams, "Physics and the Information Revolution," Physics Today, p. 38, January 2000.

41. S. Lloyd, Universal Quantum Simulators, Science, 273, 1073 (1996).

42. P. Shor, "Algorithm for Quantum Computation: Discrete Log and Factoring," Proc. $35^{\text {th }}$ Annual Symposium on Foundation of Computer Science, ed. Goldwasser, IEEE Computer Society.

43. L. K. Grover, Phy. Rev. Let., 79, 325 (1997).

44. K. Mattl, W. Weinforter, and A. Zeilinger, Phys. Rev. Let., 76, 4656 (1996).

45. A. K. Ekert, "Quantum Crypotography Based on Bell's Theorem," Phys. Rev. Let., 67, 661 (1991).

46. C. H. Bennet et al., "Teleporting an Unknown Quantum State Via Dual Classic and EPR Channels."

47. D. Bonwmeester et al., "Experimental Quantum Teleportation," Nature, 390, 575 (1997).

48. J. J. Cirac and P. Zoller, "Quantum Computation with Cold Trapped Ions," Phys. Rev. Let., 74, 4091 (1995).

49. A. Steane, "The Ion Trap Information Processor," eprint quant-ph/9608011.

50. M. Horodecki et al., Phys. Rev. Let., 80, 5239 (1998).

51. J. A. Jones and M. Mosca, J. Chem. Phys., 69, 1648 (1998).

52. H. J. Yao and J. H. Eberly, Phys. Rev. Rep., 118, 239 (1985). 
53. D. P. DiVincenzo and D. Loss, Superlattices and Microstructures, 23, 419 (1998).

54. Päivi Törmä, "Transitions in Quantum Networks," Phys. Rev. Let., 81, 2185 (1998).

55. J. Caulfield, "Prespective in Optical Computing," Computer, p. 22, February 1998.

56. R. Landauer, local citation, 39, p. 59.

57. D. DiVicenzo and B. Terhal, "Decoherence: The Obstacle to Quantum Computation," Physics World, 11, 53 (1998).

58. C. K. Ong, G. M. Huang, T. J. Tara, and J. W. Clark, Math System Theory, 17, 335 (1984).

59. M. Dahleh, A. P. Pierce, and H. Rabitz, Phys. Rev., A47, 4593 (1993).

60. A.-Luis and L. L. Sanchez-Soto, Phys. Rev. Let., 81, No. 19, p. 4031 (1998).

61. R. B. Perez, V. Protopopescu, J. L. Munoz-Cobo, Mathematical Models and Methods in Applied Sciences, 9, No. 2, 305 (1999).

62. G. Harel and V. M. Akulin, Phys. Rev. Let., 82, No. 1, 1 (1999).

63. P. Shor, "Fault Tolerant Quantum Computation, eprint quant-ph/96030.

64. D. P. DiVincenzo and P. W. Shor, "Fault Tolerant Error Correction with Efficient Quantum Codes," Phys. Rev. Let., 77, 3260 (1996).

65. C. J. van Euk, J. J. Cirac, and P. Zoller, Phys. Rev. Let., 78, 4293 (1997).

66. E. P. Wigner, "The Problem of Measurement," American Journal of Physics, 31, 6 (1963). 


\section{APPENDIX A}

Consider the $\mathrm{SU}(2)$ operator $(\mathrm{n}=2 . \mathrm{s}=4-1=3, \mathrm{r}=1)$

$$
\hat{A}=\overrightarrow{\hat{\sigma}} \cdot \vec{a}=\hat{\sigma}_{1}, a_{1}+\hat{\sigma}_{2} a_{2}+\hat{\sigma}_{3} a_{3}
$$

where $\overrightarrow{\hat{\sigma}}$ and $\overrightarrow{\mathrm{a}}$ are the vectors of components

$$
\begin{aligned}
& \hat{\sigma}_{1}=\hat{\mathrm{P}}_{12}+\hat{\mathrm{P}}_{21}: \hat{\sigma}_{2}=i\left(\hat{\mathrm{P}}_{12}-\hat{\mathrm{P}}_{21}\right) ; \hat{\sigma}_{3}=-\hat{\mathrm{P}}_{11}+\hat{\mathrm{P}}_{22} \\
& a_{1}=\sin \theta ; \mathrm{a}_{2}=0_{1} \quad \mathrm{a}_{3}=\cos \theta .
\end{aligned}
$$

To obtain the construction of this operator is terms of the SU(2) generators, $\hat{\lambda}_{1}, \hat{\lambda}_{2}, \hat{\lambda}_{3}$. given from (2.21) and (2.22) as

$$
\hat{\lambda}_{1}=\hat{\mathrm{u}}_{12}=\hat{\mathrm{P}}_{12}+\hat{\mathrm{P}}_{21} ; \hat{\lambda}_{2}=\hat{v}_{12}=i\left(\hat{\mathrm{P}}_{12}-\hat{\mathrm{P}}_{21}\right) ; \hat{\lambda}_{3}=\hat{\mathrm{w}}_{1}=-\hat{\mathrm{P}}_{11}+\hat{\mathrm{P}}_{22}
$$

we use (2.24):

$$
A_{0}=T_{r}\{\hat{A}\}=a_{1} T_{r}\left(\hat{P}_{12}+\hat{P}_{21}\right)+a_{3} T_{r}\left(-\hat{P}_{11}+\hat{P}_{22}\right)=0(\text { in view of } 2.19)
$$

next

$$
\begin{aligned}
A_{1}=\operatorname{Tr}\left\{\hat{\mathrm{A}} \hat{\lambda}_{1}\right\} & =T_{r}\left\{\mathrm{a}_{1}\left(\hat{\mathrm{P}}_{12} \hat{\mathrm{P}}_{12}+\hat{\mathrm{P}}_{12} \hat{\mathrm{P}}_{21}+\hat{\mathrm{P}}_{21} \hat{\mathrm{P}}_{12}+\hat{\mathrm{P}}_{21} \hat{\mathrm{P}}_{21}\right)\right. \\
& \left.+\mathrm{a}_{3}\left(\hat{\mathrm{P}}_{22} \hat{\mathrm{P}}_{12}+\hat{\mathrm{P}}_{22} \hat{\mathrm{P}}_{21}-\hat{\mathrm{P}}_{11} \hat{\mathrm{P}}_{12}-\hat{\mathrm{P}}_{11} \hat{\mathrm{P}}_{21}\right)\right\}
\end{aligned}
$$

use of (2.19) yields

$$
A_{1}=2 a_{1}=2 \sin \theta \text {. }
$$

In the same manner is easily checked that

$$
\mathrm{A}_{2}=0 ; \quad \mathrm{A}_{3}=2 \cos \theta
$$


This page intentionally left blank. 


\section{APPENDIX B: PROJECTION POSTULATE}

Given the ensemble described by the density operator

$$
\hat{\rho}=\sum_{i,} \rho_{i,}|i><j|
$$

find the probability. $\mathrm{p}_{11}$, of having the result. $\hat{\mathrm{P}}_{11}=|1><1|$

From Eqs. (5.9) and (B.1), we have

$$
\mathrm{p}_{11}=\mathrm{T}_{r}\left\{|1><1| \sum_{1,}\left|i>\rho_{1,}<j\right|\right\}
$$

get now the trace, and use orthogonality

$$
\mathrm{p}_{11}=\sum_{k} \rho_{k k}<1|k\rangle<k|1\rangle=\rho_{11} .
$$

The "reduction" of the wave function is seen to happen using (5.8) and (B.1)

$$
\left.\hat{\rho}^{\prime}=\frac{1}{\mathrm{p}_{11}}\left\{|1><1| \sum_{11} \rho_{i,}|i\rangle<j|| 1\right\rangle<1 \mid\right\}=\frac{1}{\mathrm{p}_{11}}\left\{\left|1>\sum_{1,} \rho_{11}<1\right| i><j \mid 1><1\right\}
$$

use orthonormality and the result (B.3), to get

$$
\hat{\rho}^{\prime}=|1><1| \text {. }
$$

Thus, after the measurement à la von Neumann one obtains the "collapsed" ensemble (B.4). 
This page intentionally left blank. 


\section{APPENDIX C: SOME ALGEBRA}

To prove the results (5.13) up to (5.15) start from Eqs. (2.24). (2.28). and (2.17)

$$
\begin{aligned}
& \hat{\mathrm{u}}_{n \prime}=\hat{\mathrm{P}}_{m}+\hat{\mathrm{P}}_{\prime \prime}: \hat{v}_{\prime \prime}=i\left(\hat{\mathrm{P}}_{m}-\hat{\mathrm{P}}_{m}\right)(\mathrm{n} \neq \mathrm{j}) \\
& \hat{w}, m>=w,{ }^{(m)}: m> \\
& \hat{\mathrm{P}}_{k}, \quad \hat{\mathrm{P}}_{m n}=\hat{\mathrm{P}}_{k n} \delta_{i m} .
\end{aligned}
$$

From (C.1) and (C.3) one obtains

$$
\hat{\mathrm{P}}_{m m} \hat{\mathrm{u}}_{n} \hat{\mathrm{P}}_{m m}=2 \hat{\mathrm{P}}_{m m} \delta_{m m} \delta_{m m}=0
$$

Same argument yields $\hat{\mathrm{P}}_{m m} \hat{\mathrm{V}}_{1 /} \hat{\mathrm{P}}_{m m}=0$. Use of Eqs. (C.2) and (C.3) yields

$$
\hat{\mathrm{P}}_{m m} \hat{\mathrm{W}}, \quad \hat{\mathrm{P}}_{m m}=\hat{\mathrm{P}}_{m m} \quad \mathrm{~W}_{i}^{(m)} \hat{\mathrm{P}}_{m m}=\mathrm{W}_{i}{ }^{(m)} \hat{\mathrm{P}}_{m m} \delta_{m m}=\mathrm{W}_{i}{ }^{(m)} \hat{\mathrm{P}}_{m m}
$$

Direct application of (C.3) yields the result (5.15). 
This page intentionally left blank. 


\section{APPENDIX D: PROJECTION ALGORITHM FOR COMPOSITE SYSTEMS}

The main mathematical manipulations to obtain the result (7.5) from Eq. (4.23) are given in this appendix. Start from the $S U(n)$ generators. $\hat{\lambda}_{1}(v)(v=1.2)$. shown in Eq. (2.23) and the relations $(5.13)$ up to $(5.15)$ that for convenience are repeated below

$$
\begin{aligned}
& \hat{\mathrm{P}}_{m m} \hat{\mathrm{u}}_{1,} \quad \hat{\mathrm{P}}_{m m !}=\hat{\mathrm{P}}_{m m m} \hat{v}_{1}, \hat{\mathrm{P}}_{m m}=0 \\
& \hat{\mathrm{P}}_{m m m} \hat{\mathrm{W}}, \hat{\mathrm{P}}_{m m n}=\mathrm{W}^{(m)} \hat{\mathrm{P}}_{m m} \\
& \hat{\mathrm{P}}_{m m} \hat{\mathrm{l}} \hat{\mathrm{P}}_{m m}=\hat{\mathrm{P}}_{m m} \text {. }
\end{aligned}
$$

Note that the projection operators above act on subsystem $\mathrm{H}(2)$. We proceed term by term in Eq. (7.4)

(1) $\rightarrow \hat{\mathrm{P}}_{m m} \hat{\mathrm{l}}(1) \otimes \hat{\mathrm{i}}(2) \hat{\mathrm{P}}_{m m}=\hat{\mathrm{i}}(1) \hat{\mathrm{P}}_{m m}(2)$

(2) $\rightarrow \hat{\mathrm{P}}_{m m} \sum_{j=1}^{1} \lambda_{j}(1)\left[\hat{\lambda}_{j}(1) \otimes \hat{\mathrm{l}}(2)\right] \hat{\mathrm{P}}_{m m}=\sum_{j=1} \lambda_{j}(1)\left[\hat{\lambda}_{,}(1) \otimes \mathrm{P}_{m m}(2)\right]$

(3) $\rightarrow \hat{\mathrm{P}}_{m+m} \sum_{j=1}^{i} \lambda_{k}(2)\left[\hat{\mathrm{l}}(1) \otimes \hat{\lambda}_{k}(2)\right] \hat{\mathrm{P}}_{m m}=\sum_{i=1}^{m-1} \mathrm{w}_{f}(2) \mathrm{w}_{i}^{(m)}\left[\hat{\mathrm{i}}(1) \otimes \mathrm{P}_{m m !}(2)\right]$.

This result arises [see Eq. (2.23)] from the fact that the elements $\hat{\mathrm{u}}_{i j}$ and $\hat{\mathrm{v}}_{1 \prime}$ of the $\hat{\lambda}_{k}(2)$ generator do not contribute to the sum, in view of (D.1). Only those componients given by the operators $\hat{\mathrm{w}}_{\ell}(\ell=1 \ldots n-1)$ do contribute according to (D.2).

$$
\begin{aligned}
(4) \rightarrow & \hat{\mathrm{P}}_{m m} \sum_{j, k}^{s} \mathrm{~K}_{j k}(1,2)\left[\hat{\lambda}_{j}(1) \otimes \hat{\lambda}_{k}(2)\right] \hat{\mathrm{P}}_{m m} \\
& =\hat{\mathrm{P}}_{m m}\left\{\sum_{j}\left\{\mathrm{~K}_{j 1}\left[\lambda_{j}(1) \hat{\mathrm{u}}_{12}(2)\right]+\ldots \mathrm{K}_{l 2}\left[\hat{\lambda}_{i}(1) \hat{\mathrm{u}}_{23}(2)\right]+\ldots\right\}\right. \\
& +\sum_{j}\left\{\mathrm{~K}_{j 1}\left[\lambda_{j}(1) \otimes \hat{\mathrm{v}}_{12}(2)\right]+\ldots \mathrm{K}_{i 2}\left\lfloor\hat{\lambda}_{j}(1) \otimes \hat{\mathrm{v}}_{23}(2)\right]+\ldots\right\} \\
& \left.+\sum_{\ell=1}^{n-1} \mathrm{~K}_{j \ell}\left[\hat{\lambda}_{j}(1) \otimes \hat{\mathrm{w}}_{i}(2)\right]\right\} \hat{\mathrm{P}}_{m m} .
\end{aligned}
$$

Again, in view of (D.1) and (D.3), one obtains

$$
\text { (4) }=\sum_{j=1}^{\infty} \sum_{i=1}^{n-1} \mathrm{~K}_{i}, \cdots+\mathrm{w}_{i}{ }^{(m)}\left[\hat{\lambda},(1) \otimes \hat{\mathrm{P}}_{m m i}(2)\right] \text {. }
$$

The second subindex in the correlation tensor $\mathrm{K}(1,2)$ is 


$$
s^{\prime}+\ell=\mathrm{n}^{2}-\mathrm{n}+\ell
$$

because of the "mutilation" of the range of the original. k. subindex due to the results $1 \mathrm{D} .11$. For instance. for $n=3 ; 1 \leq t \leq 2$. We have [see Eq. (2.23)]

$$
\overline{\hat{\lambda}}=\left\{\hat{u}_{12}, \hat{u}_{13}, \hat{u}_{23}, \hat{v}_{12}, \hat{v}_{13}, \hat{v}_{23}, \hat{w}_{1}, \hat{w}_{2},\right\}
$$

it follows that the second subindex must match the $7^{\text {th }}$ and $8^{\text {th }}$ positions of the seventh and eight components; i.e..

$$
\sum_{i=1}^{2} K_{1.0+1}=K_{1.7}+K_{1.8}:
$$

To derive the result (7.9), insert Eq. (7.7) for the projector operator $\hat{\mathrm{P}}_{\min }(2)$ into Eq. (7.5) and rearrange to get:

$$
\begin{aligned}
& p_{m}(2) \hat{\rho}^{\prime}=\frac{1}{\mathrm{n}^{2}} \hat{\mathrm{l}}(1) \otimes \hat{\mathrm{l}}(2)\left[\frac{\mathrm{l}}{\mathrm{n}}+\sum_{i=1}^{n-1} \mathrm{w}_{\ell}(2) \mathrm{w}_{f}{ }^{(m)}\right] \\
& +\frac{1}{2 \mathrm{n}} \sum_{j=1}^{n-1}\left[\frac{1}{\mathrm{n}} \lambda_{,}(1)+\frac{1}{2} \sum_{i=1}^{n-1} \mathrm{~K}_{j . n^{\prime}++} \mathrm{w}_{i}{ }^{(m)}\right]\left[\hat{\lambda}_{,}(1) \otimes \hat{\mathrm{i}}(2)\right]
\end{aligned}
$$

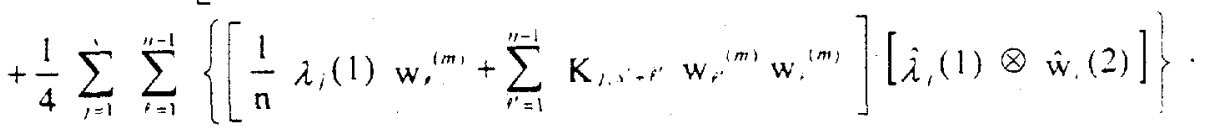

Next on account of the expression (7.8) for $\mathrm{p}_{\mathrm{m}}(2)$, one obtains dividing through by $\mathrm{p}_{\mathrm{m}}(2)$ the result (7.9) in text. 


\section{APPENDIX E: CALCULATION OF $\mathrm{C}_{A A}$}

We start from Eq. (7.35) written in the $\mathrm{SU}(2) \otimes \mathrm{SU}(2)$ formalism, where since $\vec{\lambda}(1), \vec{\lambda}(2)$ are zero and $\mathrm{K}_{\mathrm{ij}}(1,2)=-\hat{\delta}_{\mathrm{ij}}$. one gets

$$
M_{i k}=-\delta_{i k} .
$$

Then Eq. (7.35) for $C_{A_{A}}$ becomes

$$
C_{t, t}=-\frac{1}{4} \sum_{i=1}^{3} A_{i}(1) A_{i}(2) .
$$

Use of Eqs. (10.2) and (10.3) in (E.2) yields

$$
\mathrm{C}_{t .4}\left(\theta_{2} \theta_{1}\right)=-\cos \left(\theta_{2}-\theta_{1}\right) \text {. }
$$

with two limiting cases

$$
\begin{array}{lll}
\theta_{1}=\theta_{2} & \mathrm{C}_{A t}=-1 \quad \text { (anticorrelated) } \\
\theta_{1}-\theta_{2}=\frac{\pi}{2} & \mathrm{C}_{A t A}=0 & \text { (uncorrelated) }
\end{array}
$$


This page intentionally left blank. 


\section{DISTRIBUTION}

1. University of Tennessee. Knoxville

H. L. Dodds

2-25. UT-Battelle

G. T. Alley

F. C. Difilippo

W. B. Dress, Jr.

C. C. Havener

D. E. Holcomb

D. P. Hutchinson

J. M. Jansen

R. A. Kisner

J. March-Leuba

J. K. Mattingly

D. W. McDonald

J. T. Mihalczo

R. B. Perez

V. A. Protopopescu

L. L. Riedinger

J. B. Roberto

D. R. Schultz

R. W. Shaw

M. L. Simpson

T. E. Valentine

J. C. Wells

J. D. White

M. C. Wright

ORNL Laboratory Records - RC 
$=$

$=$

$a$

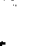

